\title{
Adaptive Antenna Pattern Notching of Interference in Synthetic Aperture Radar Data Using Digital Beamforming
}

\author{
Tobias Bollian 1,2,3,*(D), Batuhan Osmanoglu ${ }^{1}$ (D), Rafael Rincon ${ }^{1}$, Seung-Kuk Lee ${ }^{1,4}$ \\ and Temilola Fatoyinbo ${ }^{1}$ (D) \\ 1 NASA Goddard Space Flight, Greenbelt, MD 20771, USA; batuhan.osmanoglu@nasa.gov (B.O.); \\ rafael.f.rincon@nasa.gov (R.R.); seungkuk.lee@nasa.gov (S.-K.L.); lola.fatoyinbo@nasa.gov (T.F.) \\ 2 Universities Space Research Association, Columbia, MD 21046, USA \\ 3 Microwaves and Radar Institute, German Aerospace Center (DLR), 82234 Weßling, Germany \\ 4 Department of Geographical Sciences, University of Maryland, College Park, MD 20742, USA \\ * Correspondence: tobias.bollian@dlr.de
}

Received: 3 April 2019; Accepted: 24 May 2019; Published: 4 June 2019

\begin{abstract}
Radio Frequency Interference (RFI) is a growing problem in Synthetic Aperture Radar (SAR) systems as scientific motivations push the radars to lower frequencies and as more wireless services share the frequency spectrum. New, advanced SAR instruments, such as NASA's EcoSAR, DBSAR and DLR's Tandem-L mission, employ a multichannel architecture capable of Digital Beamforming (DBF). Radars with DBF are capable of notching the antenna pattern in specific directions, which can be utilized to suppress RFI on board or in post-processing. A well-researched beamformer for this purpose is the Minimum Variance Distortionless Response (MVDR) Beamformer. However, the number of interferers that can be removed through notching is limited by the number of receive channels. It is therefore essential to adaptively change the antenna pattern notching throughout the image in time and frequency for the best results with a given number of receive channels. In this paper, we present several methods to achieve this notching by making use of the spatial SAR signal distribution in range time, range frequency, azimuth time and azimuth Doppler that is inherent to the SAR imaging geometry. Because this distribution is time-variable and yet predictable, it can be used to improve the angle of arrival estimation of the RFI and the adaptive notching. The presented methods can be applied to a Digital Beamforming (DBF) SAR signal with multiple channels in elevation and/or in azimuth. Simulations show that the proposed methods increase the ability to notch out-of-swath interference from multiple directions and lessen the impact on in-swath interference. The improvement of the interferometric coherence for a single-pass interferogram acquired by NASA's EcoSAR system (capable of DBF in elevation) is demonstrated. The removal of periodic RFI artifacts is achieved.
\end{abstract}

Keywords: Synthetic Aperture Radar; interference; RFI; digital beamforming; beamformer

\section{Introduction}

Radio Frequency Interference (RFI) is a problem that is continuously growing due to the demand for wireless services and the finite nature of the frequency spectrum [1]. RFI varies locally and temporally because of different frequency regulations in different countries [2]. Allowed services in the regulated spectrum can range from communication systems to scientific instruments; television broadcast systems and military usage. In addition, the demand for certain services depends on the population density of the area. The source of the interference is therefore steadily changing and predictions about the nature of the interfering signals are complicated. This poses a major challenge 
to Synthetic Aperture Radar (SAR) systems operating at P-band and L-band [3]. This challenge begins with the degradation of polarimetric and geometric calibration [4] efforts during a missions commissioning phase if calibrations sites are impacted by RFI. Even if measures are taken to ensure the calibration sites are free of interference, RFI can emerge in the operational mode and bias the measured backscatter, corrupt polarimetric signatures and decorrelate interferometric SAR data $[5,6]$. To ensure the integrity of derived science products, the mitigation of RFI is critical.

Conventional RFI mitigation techniques can be grouped into three categories [6-9]: notching methods, subtraction methods and filter methods. Conventional notching methods remove samples from the data that are corrupted by RFI. This is done either in the time or frequency domain depending on the nature of the interference. For example, narrowband RFI can easily be notched in the frequency domain but is spread in the time domain. The advantage of notching is the fast implementation at a low computational cost. Disadvantages include SNR drop due to a loss of part of the desired signal, an inevitable distortion of the SAR Impulse Response Function (IRF) and an increase of its sidelobes [8]. The subtraction methods aim at estimating and modelling the RFI based on a priori information $[7,10,11]$. The estimated interference is then coherently subtracted without distorting the SAR signal. However, results depend on how well the assumed model fits the RFI. Filtering methods often implement adaptive filters as, for example, the Least Mean Square (LMS) filter in References [12,13]. Converging and performance of the filter rely on a careful selection of the filter parameters, which depend on the SAR-to-RFI level. Another filtering method is spatial filtering by shaping the antenna pattern.

Spatial filtering depends on the interferer's location and is independent of statistics of the interfering signal and the SAR-to-RFI ratio. This can be achieved with auxilliary antennas that act as a sidelobe canceller (SLC) [14-16]. The information that is gathered with the auxiliary antennas is coherently subtracted and the antenna pattern (AP) is nulled towards the interferer. The SLC has been investigated in the context of SAR in References [17-19]. In Reference [20], the phased array of the SAR is used for an interferer with known angle of arrival (AoA) or several auxiliary antennas are used to adapt the antenna pattern for interferer cancellation on a pulse by pulse basis. This method is be performed at the cost of the additional auxiliary antennas or the reduced size of the main antenna.

New, advanced SAR systems, such as NASA EcoSAR [21], NASA DBSAR [22] and DLR Tandem-L [23], incorporate a multichannel architecture that opens up new opportunities for filtering RFI. The individual recording of multiple subelements of an antenna array allows for digital beamforming (DBF) [24], a technique that is used to synthesize antenna patterns post-data acquisition [25]. Therefore, the interferer AoA and thus the optimal antenna pattern can be estimated from the data itself. In addition, all possible antenna patterns can be synthesized simultaneously, which eliminates the need for separate auxiliary antennas. This raises the question to what extent DBF can be used to improve RFI mitigation in SAR images and is the objective of this paper. The methods presented in this work are unique as the basis of the RFI suppression is to utilize the spatial distribution of the SAR signal that is inherent to its imaging geometry. This distribution is variable in time and yet predictable because the arrival time of the radar return is determined by the distance. The Doppler frequency of the radar return is directly related to the distance and the relative motion between the platform and the target. This can be used as a-priori information on the SAR signal and helps to characterize the unwanted RFI signals in the data. The angles of arrival of the interferers can thus be estimated for all time instances when the radar position is not identical to the instantaneous radar return position. In addition, this information can also be utilized as input for an optimal and variable antenna pattern notching throughout the image (Note that this is only possible with DBF and not with conventional phased arrays that need to use the same antenna pattern for the entire receive window). For example, in the same manner that the Scan-on-Receive (SCORE) technique $[26,27]$ is commonly used in DBF SAR to steer the main beam within the receive window, the interference cancellation can be adapted to each range line. For a DBF system with multiple channels in elevation, this can be achieved due to the aforementioned arrival time dependency of the AoA on the instantaneous 
SAR signal [26]. For a DBF system with multiple channels in azimuth however, the AoA in the range-Doppler domain can be utilized. An additional benefit of changing the antenna pattern notching within the receive window is that the strongest interfering source for each pixel can be notched independently. This minimizes the interference locally and is especially important as the number of instantaneous notches is limited by the number of receive channels.

The work is structured as follows: The theoretical background for the methods is presented in Section 2. Section 3 addresses the application of DBF for RFI mitigation in a way that utilizes the spatial distribution of the SAR signal in the range-Doppler domain. Several algorithms with varying computational work are presented for DBF SAR systems in elevation, in azimuth and in both dimensions. The potential of the newly introduced algorithms is then simulated in Section 4 for different interference scenarios and for different antenna array lengths. Section 5 is providing a verification of the previously presented algorithms for DBF in range. This verification is done by applying the algorithms to experimental airborne SAR data that is affected by RFI. The improvement of the image quality is assessed by means of the interferometric coherence of a single-pass interferogram. A conclusion is presented in Section 6.

\section{Theoretical Background}

\subsection{Minimum Variance Distortionless Beamformer}

The spatial suppression of RFI can be achieved with adaptive beamforming. Hereby, the main beam is preserved in the direction of the signal of interest (SOI) but nulls are placed in the antenna pattern towards the direction of the interferers. A well-researched adaptive beamformer for this purpose is the Minimum Variance Distortionless (MVDR) (or Capon) beamformer [25,28-30]. The preservation of the SOI is guaranteed by requiring a unity gain in its direction with

$$
\mathbf{w}^{H} \mathbf{a}\left(\theta_{\mathbf{d}}\right)=1,
$$

where $\mathbf{w}$ are the optimal beamforming weights, $\lambda$ is the wavelength, $\theta_{\mathrm{d}}$ is the AoA of the SOI and $\mathbf{a}(\theta)$ is the steering vector. The steering vector represents the phase delay between the $N$ channels for a signal impinging the antenna from direction $\theta$ and depends on the element spacing $d$ :

$$
\mathbf{a}(\theta)=\left[\begin{array}{llll}
1 & \mathrm{e}^{\mathrm{j} 2 \pi d / \lambda \sin (\theta)} & \ldots & \mathrm{e}^{\mathrm{j} 2 \pi d / \lambda(N-1) \sin (\theta)}
\end{array}\right] .
$$

Simultaneously, the signal-to-noise-plus-interference ratio is maximized by minimizing the beamformed interference-noise-covariance (INC) matrix according to:

$$
\min \mathbf{w}^{H} \mathbf{R} \mathbf{w}
$$

Here, $\mathbf{R}$ is the interference noise covariance (INC) matrix which is discussed in Section 2.2. It can be shown that the optimal weights $\mathbf{w}$ that fullfill Equations (1) and (3) can be computed via

$$
\mathbf{w}=\frac{\mathbf{R}^{-1} \mathbf{a}\left(\theta_{\mathrm{d}}\right)}{\mathbf{a}^{H}\left(\theta_{\mathrm{d}}\right) \mathbf{R}^{-1} \mathbf{a}\left(\theta_{\mathrm{d}}\right)} .
$$

A drawback of the MVDR is its sensitivity to errors of the steering vector $\mathbf{a}(\theta)$ [31-34], which can be introduced by instrument calibration errors or by inaccuracies in the knowledge of $\theta_{d}$, for example, due of topography [35]. Note that the SCORE operation of a DBF SAR is affected by the same inaccuracy of the steering vector as mentioned in Reference [35]. Hence, it is assumed for the rest of this work that $\mathbf{a}(\theta)$ is known well enough to perform SCORE and the MVDR is sufficient. However, without loss of generality, the MVDR can be substituted by the Linearly Constraint Minimum Variance (LCMV) [36] beamformer in the developed algorithms. Then, uncertainties of the AoA can be addressed by broadening the main lobe with the LCMV, which adds further constraints. The number 
of nulls that can be placed by the MVDR is $N-1$. Each additional constraint on the main lobe set by the LCMV comes with the tradeoff of one less null that can be placed in the antenna pattern.

\subsection{Interference-Noise-Covariance Estimation}

The MVDR beamformer solution of Equation (4) relies on knowledge about the INC matrix $\mathbf{R}$. Because $\mathbf{R}$ is generally not known, it needs to be approximated by calculating the sample covariance matrix over $v$ samples via

$$
\mathbf{R}_{\mathbf{x}}=\frac{1}{v} \sum_{k=1}^{v} \mathbf{x}(k) \mathbf{x}^{H}(k),
$$

where $\mathbf{x}(k)$ is the signal matrix with a number of rows equivalent to the channel count $N$. Each row is the received signal from a receive channel. However, $\mathbf{R}_{\mathrm{x}}$ can only be estimated on the received signal that is composed not only of the interference and noise but also of the SOI. As a result, the MVDR tries to minimize the SAR signal as well, which is a disadvantage. A solution for this was presented in Reference [37], where the transmission of the SAR pulse is skipped periodically or randomly. In this way, the authors estimate the INC on noise pulses in the absence of the SAR signal. This approach requires the use of frequent noise pulses in which the radar is not transmitting, so that it can adapt to a time-varying interferer environment and to the motion of the radar platform (which affects the AoA).

A new SAR technique called Staggered SAR is changing the PRF from pulse to pulse with the goal to remove blind ranges in the SAR image [38-41]. Consequently, Staggered SAR introduces missing samples, whose range position vary across the image. Staggered SAR still performes well because not more than one consecutive sample is missed at the same range. This can not be achieved with frequent noise pulses as proposed in Reference [37]. Further, pulsed radars might not affect every pulse in the image and could be missed. And especially communication signals with time bursts can lead to many different interferer constellations (and thus INC matrices) inside the same receive window. It is therefore desired to compute many INC estimates without relying on noise pulses or tight calibration windows.

In Reference [42], the authors propose a reconstruction of the INC that makes use of the capon spatial spectrum estimator [28]:

$$
P(\theta)=\frac{1}{\mathbf{a}^{H}(\theta) \mathbf{R}_{\mathbf{x}}^{-1} \mathbf{a}(\theta)} .
$$

$P(\theta)$ gives an estimate of the power that is impinging the antenna from the direction $\theta$. By integrating $P(\theta)$ over angular regions that are not occupied by the SOI, the INC can be reconstructed as shown in Reference [42]:

$$
\mathbf{R}=\int_{\bar{\Theta}} P(\theta) \mathbf{a}(\theta) \mathbf{a}^{H}(\theta) \mathrm{d} \theta=\int_{\Theta} \frac{\mathbf{a}(\theta) \mathbf{a}^{H}(\theta)}{\mathbf{a}^{H}(\theta) \mathbf{R}_{\mathbf{x}}^{-1} \mathbf{a}(\theta)} \mathrm{d} \theta .
$$

Note that $\Theta$ is the angular area occupied by the SOI. This approach is appealing for a SAR system because the AoA of the SOI, which changes with fast time and Doppler, is predetermined by the SAR imaging geometry. Therefore, in Section 3, the MVDR and the INC reconstruction of Equation (7) are expanded into multiple RFI mitigation algorithms that are directly applicable to SAR.

\subsection{The Angular Extension of SAR Signals}

The long chirps that are generally used in SAR cause every point on the ground to be illuminated by a duration equivalent to the pulse duration $T_{\mathrm{p}}$. As a consequence, target returns from multiple elevation angles are arriving at the antenna simultaneously. This instantaneous illuminated area on the ground is the pulse extent (PE) illustrated in Figure 1a, which covers the slant range distance [43]

$$
R_{\mathrm{PE}}=\frac{T_{\mathrm{p}} \mathrm{c}_{0}}{2} .
$$


Note that $c_{0}$ refers to the speed of light. The tilt of the antenna is hereby arbitrary and does not affect the DBF performance significantly. Because returns from the entire PE are received at once, the angular spectrum (Equation (6)) estimated on the raw data is occupied by the SAR signal from multiple ranges (and thus AoAs) simultaneously. This is shown in Figure $1 \mathrm{~b}$ for a complex-circular Gaussian SAR signal. The signal is simulated with a complex-circular Gaussian backscatter between $24^{\circ}$ and $60^{\circ}$ AoA look angle under the simulation conditions of Table 1 (Section 2.3). The transmit pulse duration is $20 \mu \mathrm{s}$. To reduce the PE the data can be range compressed.

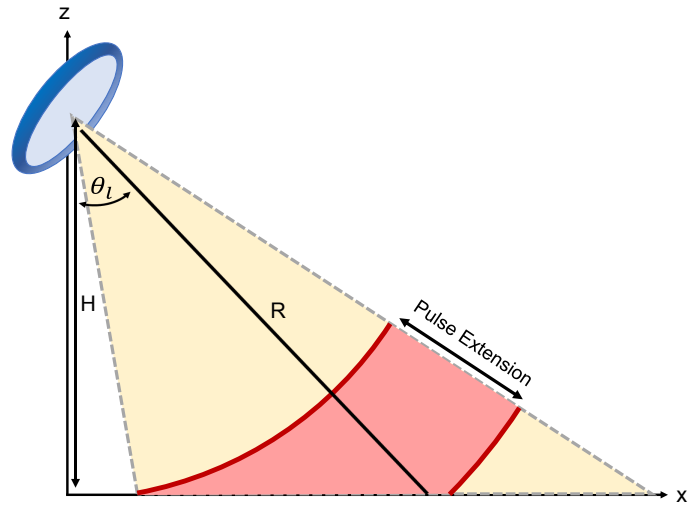

(a)

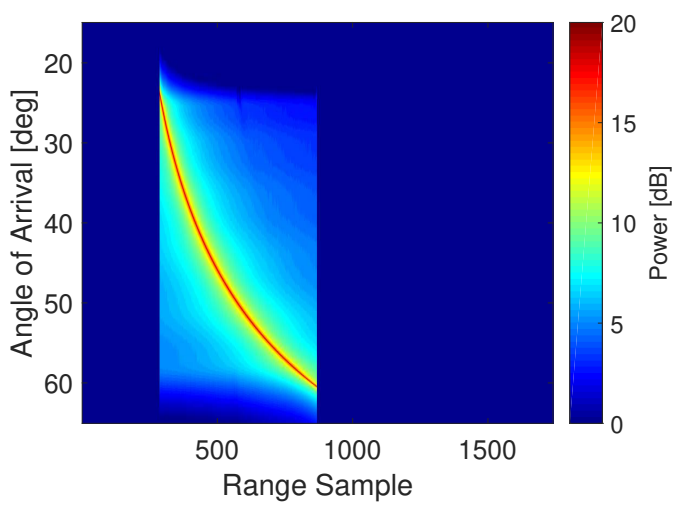

(c)

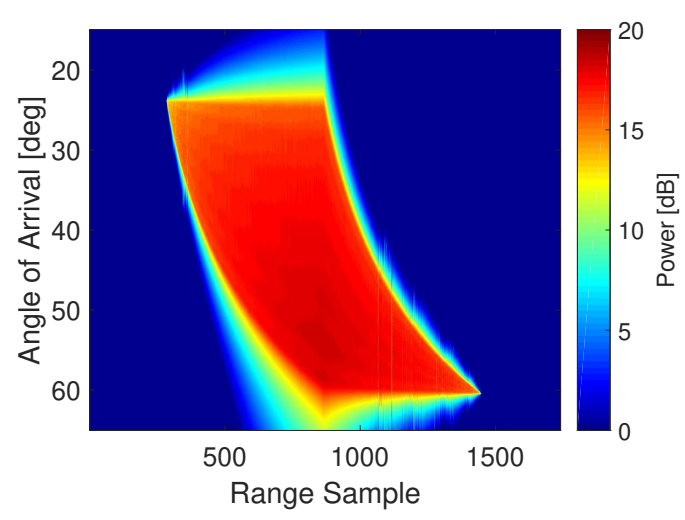

(b)

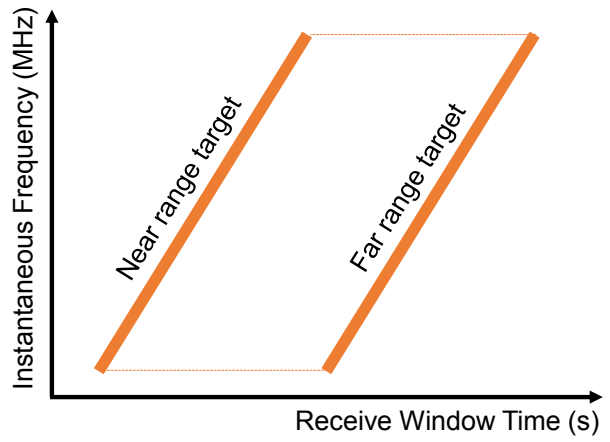

(d)

Figure 1. (a) Illustration of the travelling pulse extension in red. Returns from all targets within the red zone arrive at the radar simultaneously. (b) Capon spatial spectrum (power from AoA) versus range sample for raw SAR signal. The radar is receiving energy from a wide span of AoAs at each range because of the pulse extension. (c) Capon spatial spectrum versus range sample for range-compressed SAR signal. The energy is compressed to the instantaneous range. (d) Instantaneous frequency of near and far range target within the receive window for a transmit pulse that is modulated with a linear frequency. The signals are simulated under the simulation conditions of Table 1 (Section 2.3). 
Table 1. Parameters used for the simulation of the Synthetic Aperture Radar (SAR) and Radio Frequency Interference (RFI) data.

\begin{tabular}{cc}
\hline Parameter & Value \\
\hline Elevation Channels & 2 to 64 \\
\hline Channel Spacing & $0.5 \lambda$ \\
\hline Sample Frequency & $290 \mathrm{MHz}$ \\
\hline Center Frequency & $435 \mathrm{MHz}$ \\
\hline Pulse Bandwidth & $120 \mathrm{MHz}$ \\
\hline Pulse Duration & $20 \mu \mathrm{s}$ \\
\hline Near Range Angle & $21^{\circ}$ \\
\hline Far Range Angle & $60^{\circ}$ \\
\hline Platform Altitude & $3.2 \mathrm{~km}$ \\
\hline Number of Pulses & 500 \\
\hline Backscatter Amplitude & Normal distribution with zero mean \\
\hline Backscatter Phase & Uniform distribution from $0^{\circ}$ to $360^{\circ}$ \\
\hline SNR & 0 dB, $37.63 \mathrm{~dB}$ \\
\hline RFI Type & Continuous Wave \\
\hline
\end{tabular}

The resulting compressed PE reduces the area in the spatial spectrum in Figure 1c.

The range time dependency of the AoA that is inherent to the imaging geometry is evident. Another possibility to reduce the PE is to create frequency sublooks with bandpass filters in the frequency domain. Because the transmit chirp is modulated with a linear frequency ramp (Figure 1d), this bandpass filter in the frequency domain results in a reduction of the PE. This comes at the price of having to perform the RFI mitigation individually on each frequency sublook and therefore increases the processing time. Further implications and better options are discussed in Section 3. In the following, the spatial PE is referred to as the angular signal extent in the angular elevation spectrum.

A similar angular signal extent of the SAR signal can be observed in azimuth as well. Returns from a wide span of Doppler angles arrive simultaneously at the antenna. As illustrated in Figure 2a, the SAR is receiving multiple returns from a target on the ground (red marker) while it is moving over the target. The resulting ground extent due to this Doppler angle span is shown in Figure $2 \mathrm{~b}$. In azimuth the extent on ground depends on the opening angle $\Theta_{\mathrm{a}}$ of the aperture and the slant range distance $R_{0}$ and is equivalent to the synthetic aperture length [44]

$$
L_{\mathrm{SA}}=\Theta_{\mathrm{a}} R_{0} .
$$

Due to this relative motion, a Doppler shift is introduced, which leads to a chirp in azimuth. The Doppler shift is depending on the AoA in azimuth. Note that the color in Figure $2 b$ represents the changing Doppler and hence the AoA in azimuth. Doppler subapertures can be performed to process the wide beam on multiple narrow beams. 


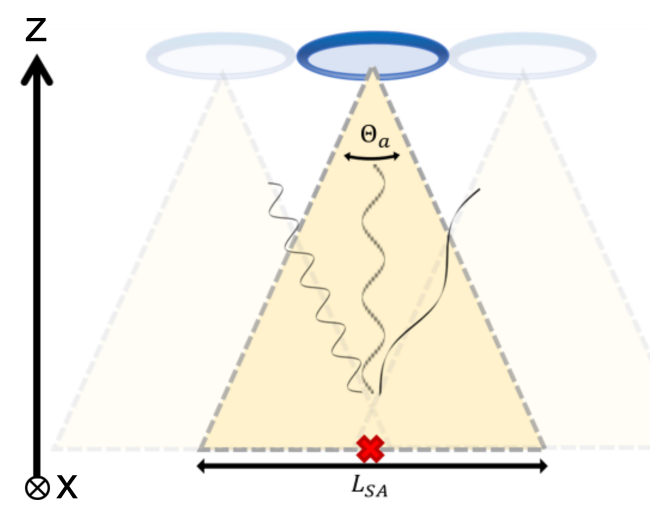

(a)

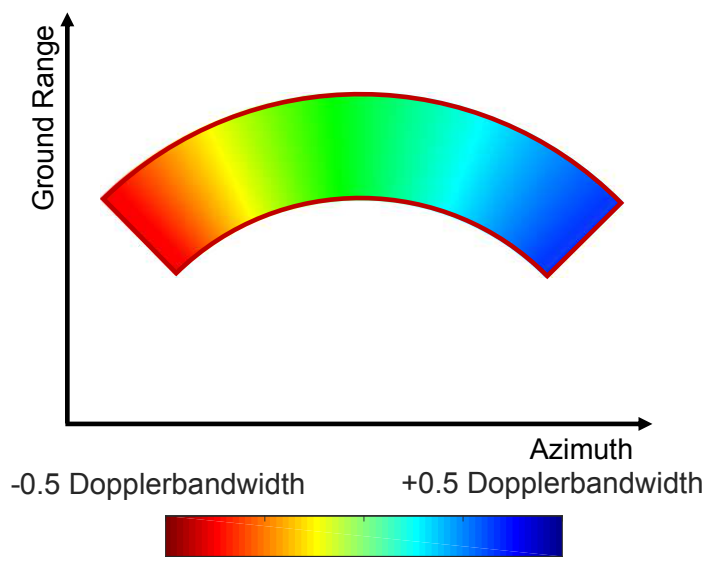

(b)

Figure 2. (a) The beamwidth in azimuth allows the SAR to record the Doppler chirp caused by the relative motion between radar and target (red mark). (b) Two-dimensional ground extent. The Doppler frequency is directly related to the azimuth AoA.

\subsection{The Impact of Range Compression on the Angular Signal Extension}

Figure 3 shows the angular signal extent $\theta_{\mathrm{ASE}}$ in elevation versus look angle $\theta_{1}$ for an airborne (orbit height $H=3 \mathrm{~km})$ and spaceborne $(H=600 \mathrm{~km})$ SAR system operating with a chirp duration of $T_{\mathrm{P}}=20 \mu \mathrm{s}$. It is evident, that the angular signal extent is especially critical in the case of an airborne system. Using a flat earth approximation (for the computation of the spaceborne scenario see Reference [43]), the angular extent is given by

$$
\left.\theta_{\mathrm{ASE}}\left(\theta_{1}\right)\right|_{\Delta r=\frac{T_{\mathrm{P}} c_{0}}{2}}=\arccos \left(\frac{H \cos \theta_{1}}{H+\Delta r, \cos \theta_{1}}\right)-\theta_{1}
$$

where $\Delta r$ refers to the slantrange extent of the pulse. It can be concluded that $\theta_{\text {ASE }}$ becomes zero for infinitely large $H$ and approaches $\theta_{\mathrm{ASE}}=90^{\circ}-\theta_{1}$ for small $H$.

By performing a range-compression, as proposed in this paper, $\theta_{\mathrm{ASE}}$ can be reduced (green line in Figure 3) to below one degree, as is the case for the spaceborne scenario (blue line) without range compression. In the same manner, the spaceborne signal could be range-compressed for a further reduction. However, this poses a challenge for the on-board processing. 


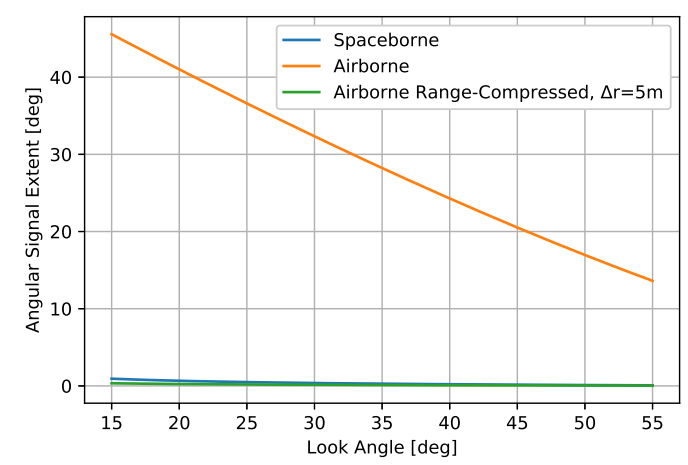

Figure 3. Angular signal extent in elevation for SAR systems operating with a 20 microsecond chirp at $H=3 \mathrm{~km}$ (airborne, orange), at $H=600 \mathrm{~km}$ (spaceborne. The green line represents the airborne system after range compression.

\section{Proposed RFI Mitigation Algorithms Using DBF}

In this section, newly developed RFI removal algorithms, which make use of the spatial distribution of the SAR signal, are presented. They are based on the MVDR beamformer with an INC estimation according to Equation (7). Thus, the INC is estimated by integrating over the angular spectrum $\Theta$ that is not occupied by the instantaneous SAR return $\Theta$ (Figure 4).

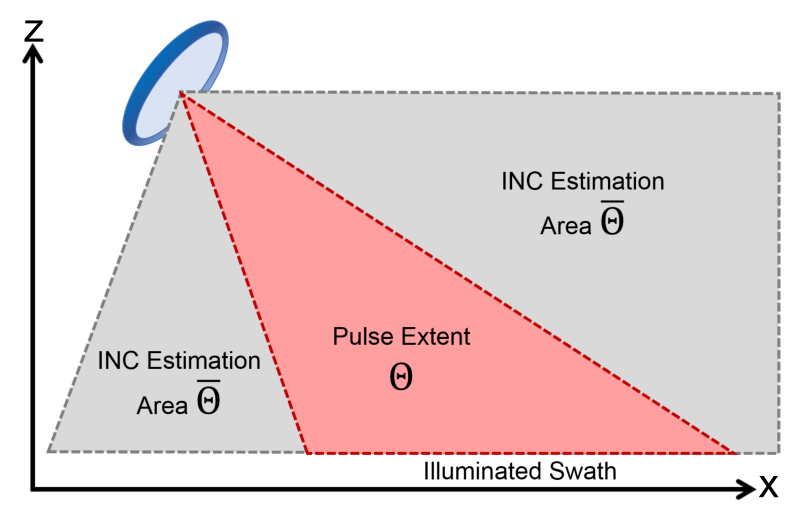

Figure 4. The estimation of the interference noise covariance (INC) matrix in the presence of the SAR signal is achieved by integrating the angular spectrum over the angular area $\bar{\Theta}$. The instantaneous SAR signal occupies the angular area $\Theta$.

As a result, the angular signal extent is generating a blindspot. All interfering sources that are in this angular area can not be detected.

This raises the question if the $\theta_{\text {ASE }}$ of a spaceborne geometry does require a further reduction that can be achieved with range compression. After the angular spectrum estimation, the SAR signal will occupy an angular area $\Theta_{\text {est }}$ equivalent to $\theta_{\mathrm{ASE}}$ plus half the angular resolution $\delta \theta_{\mathrm{el}}$ of the spectrum estimator on both sides. Thus,

$$
\max \left(\Theta_{\text {est }}\left(\theta_{1}\right)\right)-\min \left(\Theta_{\text {est }}\left(\theta_{1}\right)\right)=\theta_{\text {ASE }}\left(\theta_{1}\right)+\delta \theta_{\text {el }},
$$

which sets a limit for separating the instantaneous SAR signal from surrounding interferers.

Now, a conservative approach for the antenna pattern notching would be to only place nulls outside of the instantaneous main lobe. Even though a notching inside the outer parts of the main lobe is possible, this requires a very good instrument calibration. Otherwise, the gain the main peak can not be predicted very well and this will degrade the radiometric calibration and increase the sensitivity to mispointing. Therefore this approach is selected in this paper. The beam width depends 
on the antenna length $L_{\mathrm{e}}$ in elevation and on the antenna length $L_{\mathrm{a}}$ in azimuth. $\Theta_{\mathrm{BW}}$ in elevation can be approximated for a linear array with $N$ elements to Reference [44]:

$$
\Theta_{\mathrm{BW}} \approx \frac{\lambda}{L_{\mathrm{e}}}=\left.\frac{\lambda}{N d}\right|_{d=0.5 \lambda}=\frac{2}{N}
$$

And thus:

$$
\theta_{\mathrm{ASE}}+\delta \theta_{\mathrm{el}}<\theta_{\mathrm{BW}}
$$

Note that $\delta \theta_{\mathrm{el}}$ depends on the used spectral estimator, the antenna length, $N$, the $S N R$ and the number of samples $v$ used for the estimation. More complex spatial estimators [45-47] better resolve signals in the angular domain but come with the cost of an increased processing. Nevertheless, this suggests that a further range compression for a spaceborne system were only required, if a very narrow beamwidth or a very long transmit chirp is used. A narrow beamwidth requires the use of dispersive beams (frequency sublooks) to compensate the pulse extension loss [43]. This results in an inherent reduction of $\theta_{\mathrm{ASE}}$, as the pulse duration will be split among the frequency sublooks.

In the following, algorithms of three different categories are presented. First, DBF algorithms for a system with $N$ channels in elevation are presented in Section 3.1. Next, they are adapted for systems with $M$ channels in azimuth in Section 3.2. Without loss of generality, algorithms from both categories can be selected and combined for a system that is capable to perform DBF in both dimensions. This allows for a two-dimensional integration of the INC and can reduce the blindspot further. However, applying both categories to a system with $M N$ channels in a sequential manner impairs the effectiveness of the algorithms ( $N-1$ are set in range first and then $M-1$ in azimuth, which results in a suboptimal performance of the MVDR). The last category (Section 3.3) describes a method for two-dimensional MVDR beamforming that is able to place a total of $(N-1)(M-1)$ notches.

\subsection{DBF in Elevation}

\subsubsection{Pulse-Wise MVDR}

The fastest approach is to estimate the INC on a pulse-wise basis in the time domain as shown in Figure 5 . Let the received signal be $s_{R}(u, p, m)$, where $u$ is the range sample, $p$ the pulse number and $m$ the channel number. For each pulse $p$ of the received signal, the sample covariance of Equation (5) is computed on

$$
\mathbf{x}_{p}(u, m)=s_{\mathrm{R}}(u, p, m) \text {. }
$$

The result is inserted into Equation (7) for

$$
\theta_{1}\left(u_{\min }\right)-\frac{\delta \theta_{\mathrm{el}}}{2} \leq \Theta \leq \theta_{1}\left(u_{\max }\right)+\frac{\delta \theta_{\mathrm{el}}}{2} .
$$

Note that $\theta_{1}(u)$ represents the look angle for range sample $u$. Next, the pulse-dependent weights $\mathbf{w}_{p}$ are calculated with Equation (4). Each pulse is then individually beamformed with $\mathbf{w}_{p}$.

A faster computation can be achieved by recalculating the beamform weights only every other pulse. However, this makes the filter vulnerable to pulsed RFI that is not present in each pulse.

Regardless of the choice, the estimation of the INC can not be computed in the entire illuminated swath, which is equivalent to the angular area $\Theta$ occupied by the angular extent of all ground returns (Figure 4). Hence, the Pulse-Wise MVDR (PWMVDR) is blind to interferers located inside the SAR swath. It's main purpose is the mitigation of out-of-swath interference. This only makes the PWMVDR a good choice for acquisitions in forest or isolated areas where no interference is expected in the imaged area itself. Images of urban areas or of areas that are expected to be contaminated by interference (e.g., an airport in the image) can not be efficiently filtered. A total of $N-1$ notches are fixed for the entire pulse to minimize the interference on a global scale. Depending on the interference, especially for a number of interferers close to or larger than $N-1$, this can limit the performance. 


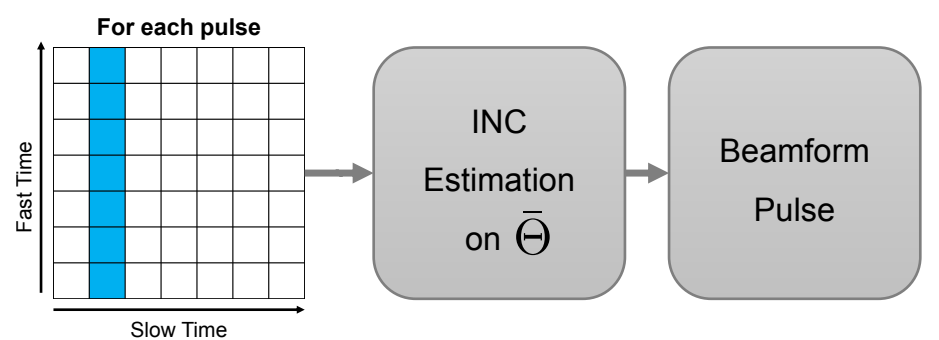

Figure 5. Pulse-Wise Minimum Variance Distortionless Response (MVDR) RFI mitigation with DBF.

A mitigation of $N-1$ interferers per pulse is possible.

\subsubsection{Segment-Wise Frequency MVDR}

A similar approach to the Pulse-Wise MVDR can be implemented in the range frequency domain. The following algorithm is depicted in Figure 6. First, a range FFT is performed on the data. Let the range-frequency azimuth-time signal of the previously used receive-signal be described with $S_{\mathrm{R}}(U, p, m)$, where $U$ represents the range-frequency bins. Then, the INC is estimated for each frequency bin $U$ to

$$
\mathbf{x}_{U}(p, m)=S_{\mathrm{R}}(U, p, m) \text {. }
$$

This requires the algorithm to be performed on a small segment of multiple pulses. Again, the result is inserted into Equation (7) for

$$
\theta_{\mathrm{l}}\left(u_{\min }\right)-\frac{\delta \theta_{\mathrm{el}}}{2} \leq \Theta \leq \theta_{1}\left(u_{\max }\right)+\frac{\delta \theta_{\mathrm{el}}}{2} .
$$

Each frequency bin line is then beamformed with the MVDR weights $\mathbf{w}_{U}$. Because each frequency bin is filtered with individual beamforming weights, the Segment-Wise Frequency MVDR (SWFMVDR) is able to place $N-1$ fixed notches at each frequency bin. Hence, for a total number of $F$ frequency bins, the total number of notches in the segment is $(N-1) F$. However, as before, the angular spectrum contains the information of the entire angular ground extent. The SWFMVDR is also blind to interferers in the entire illuminated swath (Figure 4) because the entire pulse was used for the FFT. Therefore, it addresses out-of-swath interferers. The advantage of this approach is that the notches are variable in the frequency domain. An image that is contaminated with many narrow band interferers (e.g., more than $N-1$ ) benefits from this approach over the Pulse-Wise MVDR.

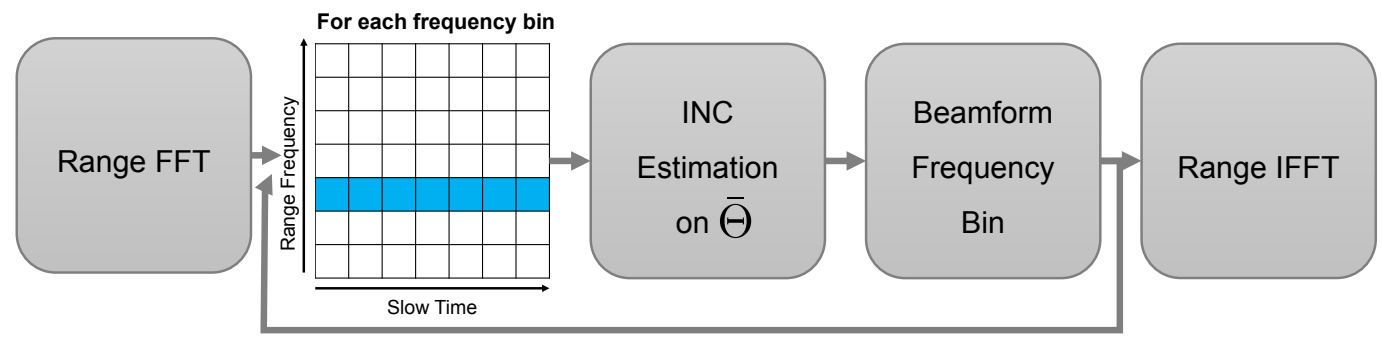

Figure 6. Segment-Wise Frequency MVDR RFI mitigation with Digital Beamforming (DBF).

A mitigation of $N-1$ interferers per frequency bin is possible.

\subsubsection{Range-Dependent Time MVDR}

It is important to reduce the blind spot of the INC estimation (angular extent of SAR signal) if the RFI mitigation is to be improved. Instead of performing pulse-wise operations, the INC estimation can be performed for different range lines. This reduces the blind spot to the angular extent of the instantaneous SAR signal, which, in elevation, is defined by the PE. Figure 7 illustrates the reduction of the blind spot. The advantage over Figure 4 is clearly visible.

As discussed in Sections 2.3 and 2.4, an airborne system benefits of an additional range compression of the data prior to the INC estimation. For a spaceborne system, this is generally 
not necessary and thus the range compression and range decompression steps applied in the following algorithms can be omitted. As shown in Figure 8, the INC can then be estimated on each range line of a small azimuth segment with

$$
\mathbf{x}_{u}(p, m)=s_{\mathrm{R}}(u, p, m)
$$

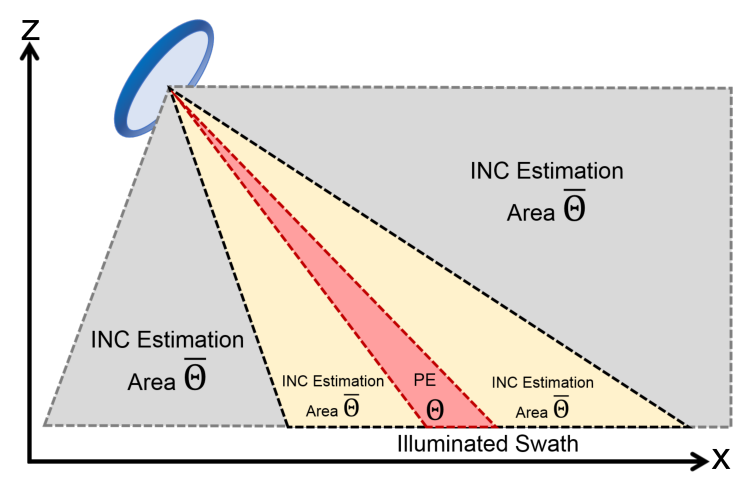

Figure 7. After range compression, the pulse extent (PE) in each range line is reduced to the resolution of the Impulse Response Function (IRF).

The number of pulses in the segment needs to be small enough to keep the AoA change of the interferer (caused by the relative motion) small, otherwise multiple notches need to be placed towards the same interferer to widen the notch. On the other hand, the estimate accuracy of the INC increases with the number of pulses. The angular integration area for the INC estimation is adaptively changed in range to the inverse of

$$
\theta_{1}(u)-\frac{\delta \theta_{\mathrm{el}}}{2} \leq \Theta \leq \theta_{1}(u)+\frac{\delta \theta_{\mathrm{el}}}{2} .
$$

Thus, the instantaneous blindspot for each line is reduced to the resolution of the IRF (if range-compressed) and depends on the transmit chirp parameters. Each range line is individually beamformed with $\mathbf{w}_{u}$. If needed, a decompression in range can now be performed. The number of instantaneous notches is $N-1$ but the notches are variable in range. This results in a local minimization of the interferer energy. In addition, the Range-Dependent Time MVDR (RDTMVDR) is also capable of automatically notching range-dependent ambiguities and orthogonal waveforms.

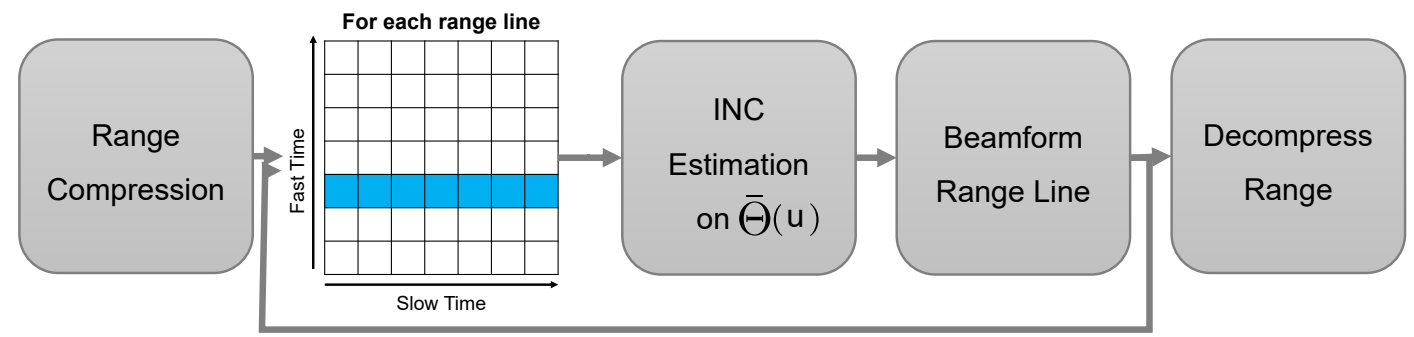

Figure 8. Range-Dependent Time MVDR RFI mitigation with DBF. A mitigation of $N-1$ interferers per range line $u$ is possible.

\subsubsection{Range-Dependent Frequency MVDR}

An extension of the Range-Dependent Time MVDR is the Range-Dependent Frequency MVDR (RDFMVDR) shown in Figure 9. Here, the raw data can be range-compressed as well to reduce the PE (e.g., necessary for the airborne case as discussed in Section 2.4). Instead of performing an INC estimation for each range line, the Range-Dependent Frequency MVDR is computing the range FFT on small and non-overlapping range windows of length $S$ (e.g., $S=64)$ from $u_{\text {start }}$ to $u_{\text {end }}=u_{\text {start }}+S$. This is illustrated with the blue arrow in Figure 9. The INC estimation is then performed for each frequency bin $U$ of each small range window on 


$$
\mathbf{x}_{u_{\text {start }}, U}(p, m)=\operatorname{FFT}\left\{\left.s_{\mathrm{R}}(u, p, m)\right|_{u_{\text {start }} \leq u \leq u_{\text {end }}}\right\}(U, p, m) .
$$

Hereby, the angular extent occupied by the instantaneous SAR signal is given by

$$
\theta_{1}\left(u_{\text {start }}\right)-\frac{\delta \theta_{\mathrm{el}}}{2} \leq \Theta \leq \theta_{1}\left(u_{\mathrm{end}}\right)+\frac{\delta \theta_{\mathrm{el}}}{2} .
$$

Next, each frequency bin line is beamformed (yellow arrow in Figure 9) with the weights $\mathbf{w}_{u_{\text {start }},} U$ and a range IFFT is performed (red arrow in Figure 9). The steps are then repeated for the next range window (gray arrow in Figure 9). In the end, the beamformed data can be decompressed in range again.

The Range-Dependent Frequency MVDR has an increased computational load compared to the Range-Dependent Time MVDR but the utilization of the frequency domain gives it the ability to place $(N-1) S$ antenna pattern notches in each range line. This is an advantage if multiple interferers are spread over the frequency spectrum and thus can be notched separately with a frequency separation. Note that the separation capabilities are limited due to the short window size and resulting spectral leakage effects [48]. An increase of the FFT window in range reduces spectral leakage but increases the blind spot, because multiple range lines and thus a larger angular extent is occupied by the instantaneous SAR signal. The full potential of the Range-Dependent Frequency MVDR can be utilized with a wideband SAR system. The advantage of the Range-Dependent Frequency MVDR over a conventional subband processing with range sublooks is discussed in the next subsection.

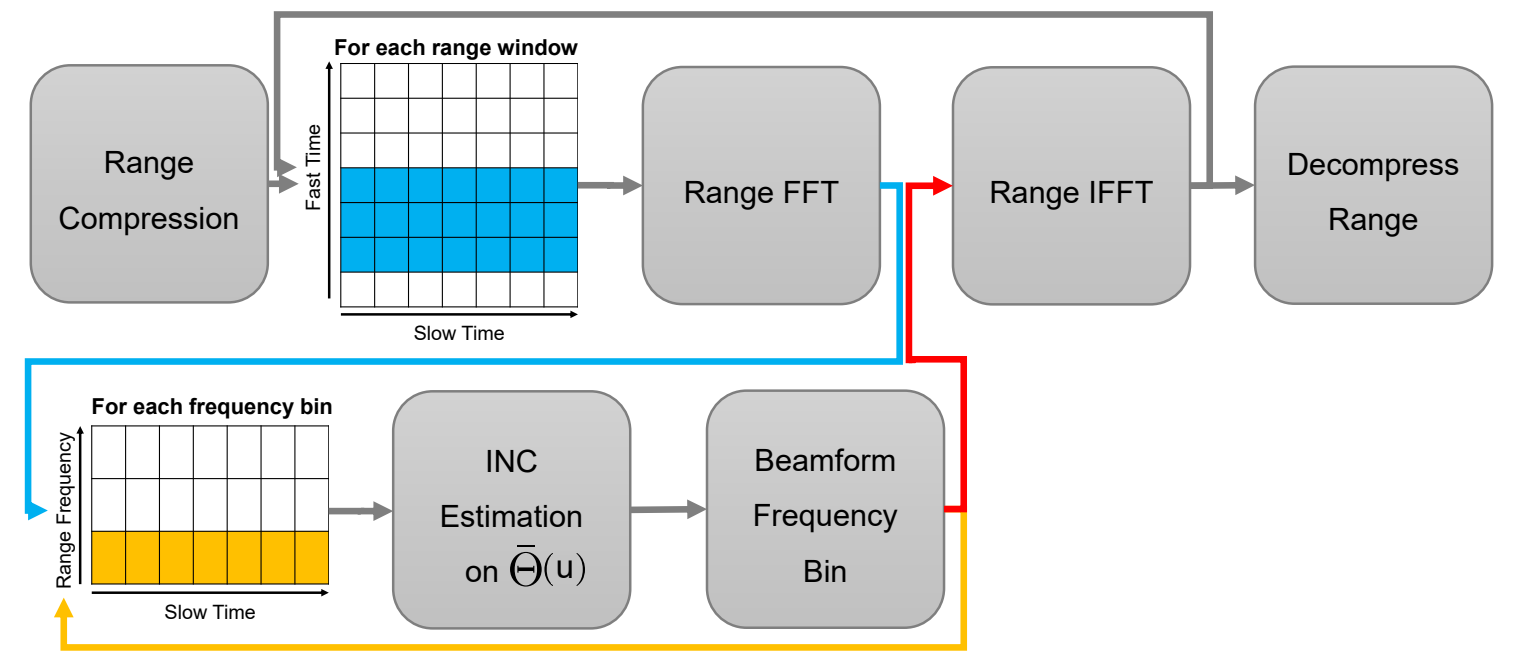

Figure 9. Range Dependent Frequency MVDR RFI mitigation with DBF. A mitigation of $N-1$ interferers per range window and frequency bin is possible. Range window is centered around range sample $u$.

\subsubsection{On the Utilization of Range-Frequency Sublooks}

Instead of applying the Range Dependent Frequency MVDR, one could also use the Range Dependent Time MVDR on multiple range-frequency sublooks. Both approaches are capable of spreading the antenna pattern notches in range and in frequency. The difference between both methods is the resulting instantaneous PE. As before, let $S$ be the number of frequency bins for the Range Dependent Frequency MVDR. In addition, we represent the number of sublooks for the Range Dependent Time MVDR with $S$ as well for the following comparison. Consequently, both algorithms are capable of placing a total of $(N-1) S$ antenna pattern notches, with $N-1$ notches per subband. 
This is beneficially in case the RFI sources are spread over the frequency domain. The slant range resolution of the focused image is approximated by Reference [44]

$$
\delta_{\mathrm{e}}=\frac{k_{0} c_{0}}{2 B_{\mathrm{W}}} .
$$

Note that $B_{\mathrm{W}}$ represents the chirp bandwidth of the entire received chirp before sublook processing. $k_{0}$ is a scaling factor set by the amplitude weighting in the frequency domain. For a rectangular window, $k_{0}=0.89$ [44]. Thus, the instantaneous PE with $S$ range-compressed sublooks is

$$
P E_{\text {sub }}=S \delta_{\mathrm{e}} .
$$

The instantaneous PE for the current range window of the Range Dependent Frequency MVDR with length $S$ is given by

$$
P E_{R D F}=\delta_{\mathrm{e}}+S \frac{c_{0}}{2 f_{\mathrm{s}}} .
$$

$f_{\mathrm{s}}$ is the sampling frequency of the data. It is assumed that $k_{0}$ is identical in both cases.

The Range Dependent Frequency MVDR is superior if the instantaneous PE is smaller:

$$
P E_{\text {sub }}-P E_{R D F}>0
$$

Inserting Equations (23) and (24) into Equation (25) yields

$$
S>\frac{1}{1-\frac{c_{0}}{2 f_{\mathrm{s}} \delta_{\mathrm{e}}}}=\frac{1}{1-\frac{B_{W}}{f_{\mathrm{s}} k_{0}}}
$$

for

$$
\frac{f_{\mathrm{s}} k_{0}}{B_{\mathrm{W}}}>1 .
$$

In case of the experimental data that is presented in Section 5, the Range Dependent Frequency MVDR is superior for $S>1.86$.

\subsubsection{Pulsed-RFI MVDR}

Interferers that transmit continuosly during the flyby time of the radar can be mitigated with the previous algorithms. However, RFI signals that appear as short pulses in the SAR data benefits from small INC estimation windows in range (Figure 10). Similar to the Range-Dependent Time MVDR, the INC estimation is performed on range windows but on each individual pulse for

$$
\mathbf{x}_{u_{\text {start }, p}}(u, m)=\left.s_{\mathrm{R}}(u, p, m)\right|_{u_{\text {start }} \leq u \leq u_{\text {end }}} .
$$

Hereby, the angular extent occupied by the instantaneous SAR signal is given by

$$
\theta_{\mathrm{l}}\left(u_{\mathrm{start}}\right)-\frac{\delta \theta_{\mathrm{el}}}{2} \leq \Theta \leq \theta_{\mathrm{l}}\left(u_{\mathrm{end}}\right)+\frac{\delta \theta_{\mathrm{el}}}{2} .
$$




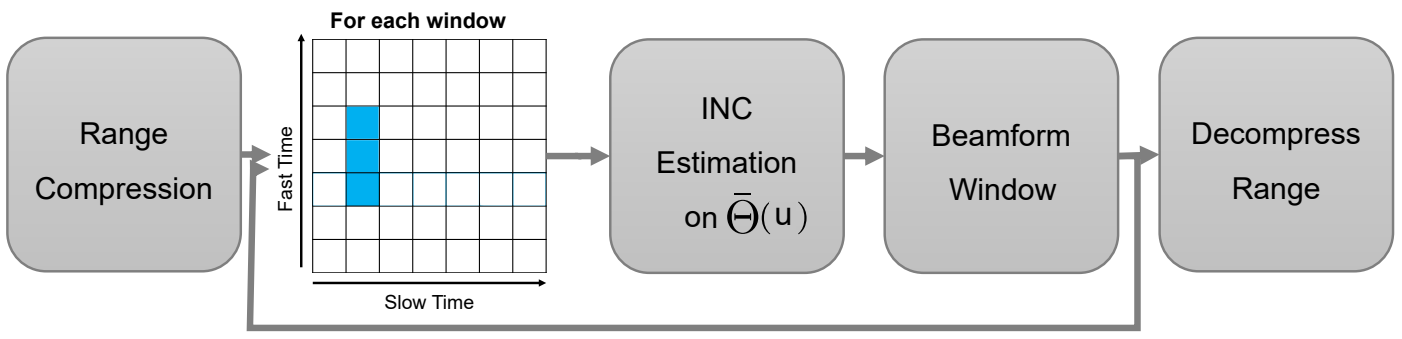

Figure 10. A Pulsed-RFI MVDR mitigation with DBF. A mitigation of $N-1$ interferers per window is possible.

\subsection{DBF in Azimuth}

In the case of a SAR with $M$ channels in azimuth, the Pulse-Wise MVDR and Segment-Wise Frequency MVDR are also applicable but limited to the same constraints. Instead of being blind in the PE extending into the range direction, the filter is blind over the Doppler angle extent in azimuth (Figures $2 \mathrm{~b}$ and 11a). Unlike before, an azimuth compression is not useful as each focused point is containing information from all AoA over the synthetic aperture. Instead, the data is converted into the range-Doppler domain because the AoA is correlated with the Doppler frequency. The resulting Doppler frequency in each column is occupying its unique AoA as illustrated in Figure 11b. Note that the PE in range is not relevant to a system with DBF in azimuth as it can not distinguish elevation angles. Two different algorithms that utilize the spatial distribution of the SAR signal in the Doppler plane are presented.
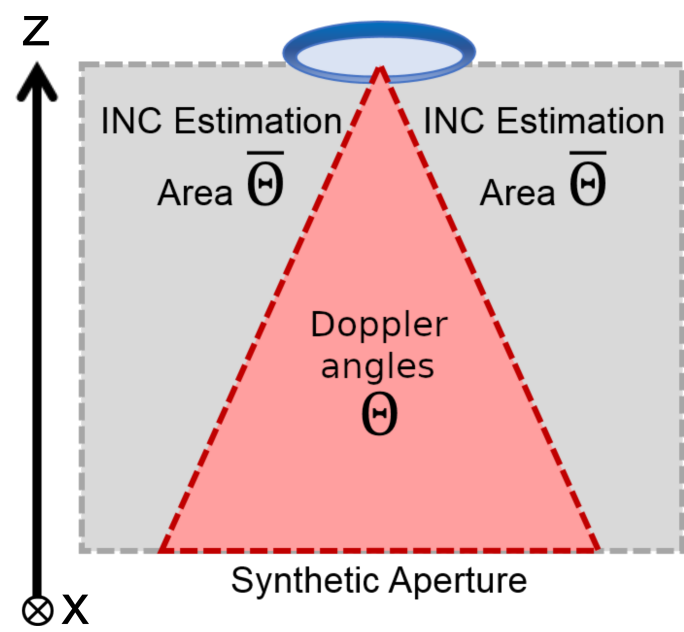

(a)

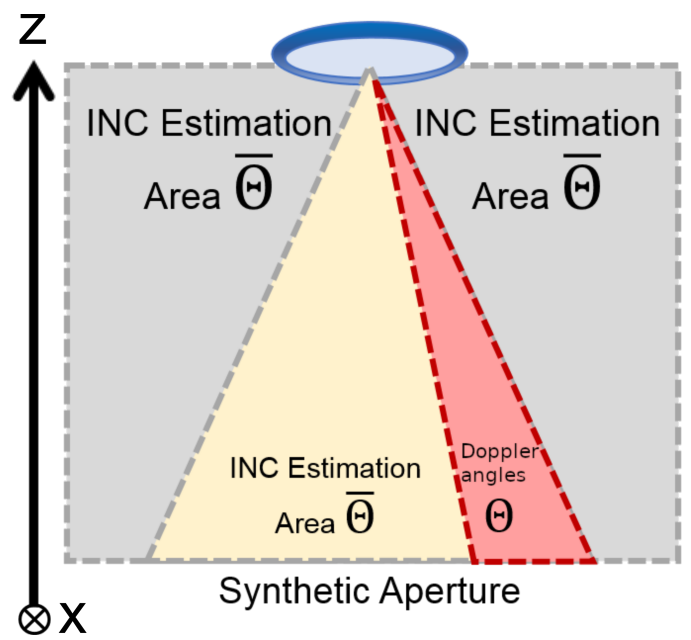

(b)

Figure 11. (a) At each time instance, the radar is receiving returns from the entire synthetic aperture. (b) In the range-Doppler domain, the AoA in azimuth is correlated with the Doppler frequency. This can be utilized to reduce the blindspot because the instantaneous Doppler angle is different in each Doppler bin.

\subsubsection{Doppler-Dependent MVDR}

For the Doppler-Dependent MVDR (DDMVDR), the data is first converted to the range-Doppler domain via an azimuth FFT as shown in Figure 12. This is performed on small azimuth segments and not on the entire image. Processing in chunks (e.g., 500 pulses) allows to adjust to a slow-time dependency of the RFI because of the platform motion. The data in each column represents a separate Doppler frequency. The INC estimation is thus performed column-wise on

$$
\mathbf{x}_{f_{\mathrm{D}}}(u, m)=\operatorname{FFT}\left\{s_{\mathrm{R}}(u, p, m)\right\}\left(u, f_{\mathrm{D}}, m\right) .
$$


Note that $f_{\mathrm{D}}$ refers to the Doppler frequency and $\theta_{\mathrm{D}}$ to the Doppler angle. The Doppler angle extent in azimuth is changed adaptively according to the known imaging geometry so that

$$
\theta_{\mathrm{D}}\left(f_{\mathrm{D}}\right)-\frac{\delta \theta_{\mathrm{az}}}{2} \leq \Theta \leq \theta_{\mathrm{D}}\left(f_{\mathrm{D}}\right)+\frac{\delta \theta_{\mathrm{az}}}{2} .
$$

Note that $\delta \theta_{\mathrm{az}}$ represents the angular azimuth resolution in this case. Each column is beamformed and an azimuth IFFT is applied. The algorithm is the equivalent to the Range-Dependent Time MVDR but adapted to the Doppler plane for a system with DBF in azimuth.

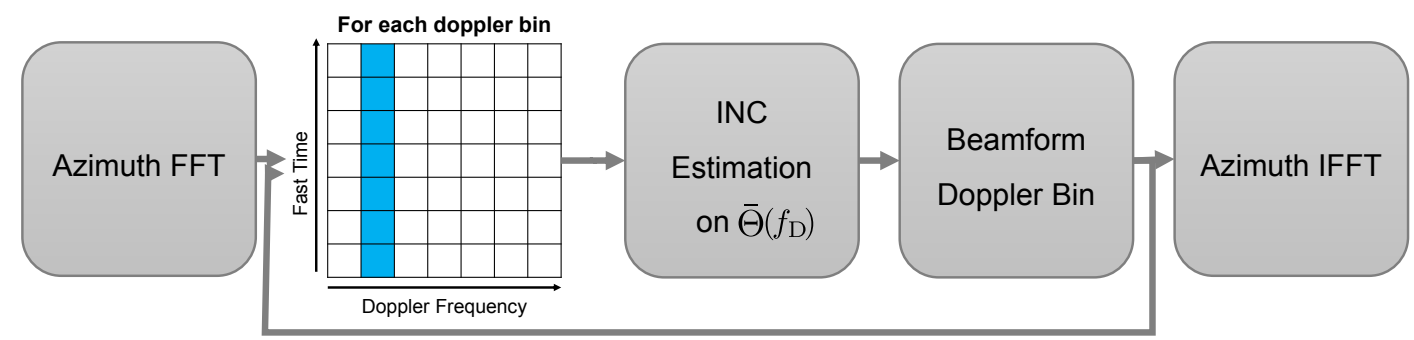

Figure 12. Doppler-Dependent MVDR RFI mitigation with DBF. A mitigation of $M-1$ interferers per Doppler bin is possible.

\subsubsection{Doppler-Dependent Frequency MVDR}

An additional range FFT is applied for the Doppler-Dependent Frequency MVDR (DDFMVDR). As displayed in Figure 13, the resulting two-dimensional frequency image chunk is then beamformed in individually windows on

$$
\mathbf{x}_{U, f_{\mathrm{D}}}\left(U, f_{\mathrm{D}}, m\right)=\operatorname{FFT} 2 \mathrm{D}\left\{s_{\mathrm{R}}(u, p, m)\right\}\left(U, f_{\mathrm{D}}, m\right)
$$

for

$$
U_{\text {start }} \leq U \leq U_{\text {end }}, f_{\mathrm{D}, \text { start }} \leq f_{\mathrm{D}} \leq f_{\mathrm{D}, \text { end }}
$$

Again, the Doppler extent in azimuth is changed adaptively according to the known imaging geometry so that

$$
\theta_{\mathrm{D}}\left(f_{\mathrm{D}, \text { start }}\right)-\frac{\delta \theta_{\mathrm{az}}}{2} \leq \Theta \leq \theta_{\mathrm{D}}\left(f_{\mathrm{D}, \text { end }}\right)+\frac{\delta \theta_{\mathrm{az}}}{2} .
$$

The dimensions of the window can be choosen at will but represent a trade off between: (a) the sample number for the INC estimation, (b) the frequency resolution of the window and (c) the Doppler resolution and hence the blindspot of the algorithm. In the end, the beamformed data is converted back to the time domain. The increased processing gives the ability to change the notches adaptively over the bandwidth of the range chirp.

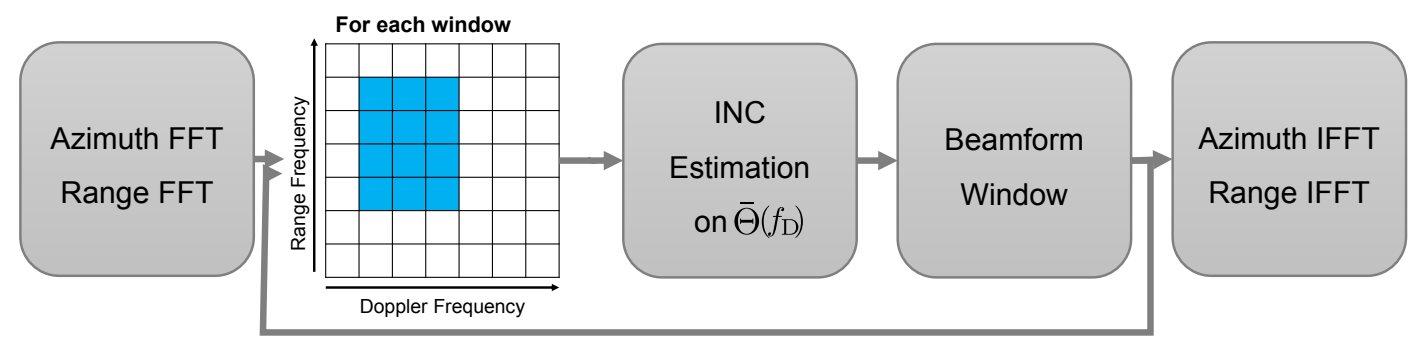

Figure 13. Doppler-Dependent Frequency MVDR RFI mitigation with DBF. A mitigation of $M-1$ interferers per window is possible.

\subsection{Two-Dimensional DBF}

A two-dimensional antenna array with $N$ channels in elevation and $M$ channels in azimuth consists of $N M$ channels. It is therefore possible to place $(N-1)(M-1)$ notches into the antenna 
pattern at the cost of the increased computational load. In addition, the two-dimensional angular resolution of the targets allows a significant reduction of the PE and Doppler angle extent. The angular extent of the ideal point target, shown in Figure 14 in yellow, can be reduced to a small patch via a range compression (e.g., necessary for the airborne case as discussed in Section 2.4) and conversion to the range-Doppler domain.

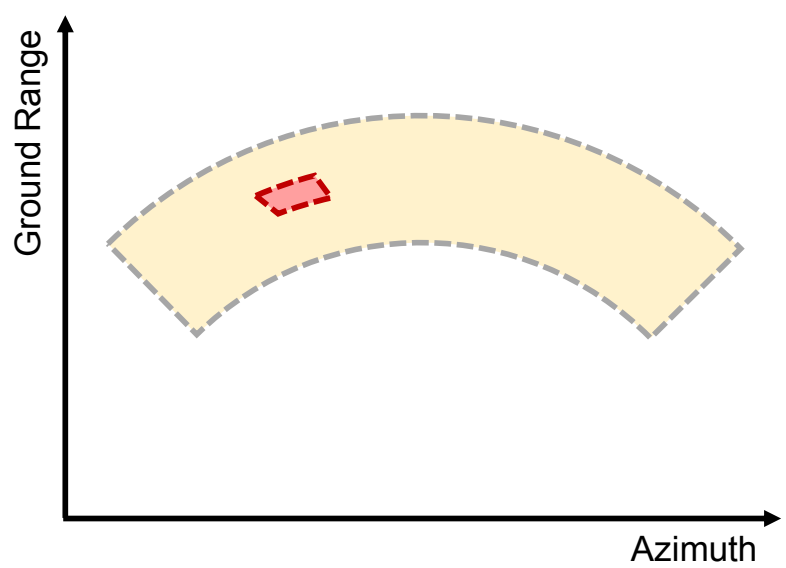

Figure 14. The pulse and Doppler extent of an ideal point target projected onto the ground (yellow). Two-dimensional DBF is capable of separating the extent to small patches (red box) in the range-compressed Doppler domain. This reduces the blindspot of the INC estimation.

The RFI filtering can then be applied with a two-dimensional MVDR (2DMVDR), shown in Figure 15. The INC estimation is applied to multiple windows

$$
\mathbf{x}_{u, f_{\mathrm{D}}}\left(u, f_{\mathrm{D}}, m\right)=\operatorname{FFT}\left\{s_{\mathrm{R}}(u, p, m)\right\}\left(u, f_{\mathrm{D}}, m\right)
$$

for

$$
u_{\text {start }} \leq u \leq u_{\text {end }}, f_{\mathrm{D}, \text { start }} \leq f_{\mathrm{D}} \leq f_{\mathrm{D} \text {,end }} .
$$

The windows are then beamformed. Again, the dimensions of the window are a trade off between instantaneous angular extent and sample size for the INC estimation. The integration is limited in both dimensions by

$$
\theta_{1}\left(u_{\text {start }}\right)-\frac{\delta \theta_{\mathrm{el}}}{2} \leq \Theta \leq \theta_{1}\left(u_{\text {end }}\right)+\frac{\delta \theta_{\mathrm{el}}}{2}
$$

and

$$
\theta_{\mathrm{D}}\left(f_{\mathrm{D}, \text { start }}\right)-\frac{\delta \theta_{\mathrm{az}}}{2} \leq \Theta \leq \theta_{\mathrm{D}}\left(f_{\mathrm{D}, \mathrm{end}}\right)+\frac{\delta \theta_{\mathrm{az}}}{2} .
$$

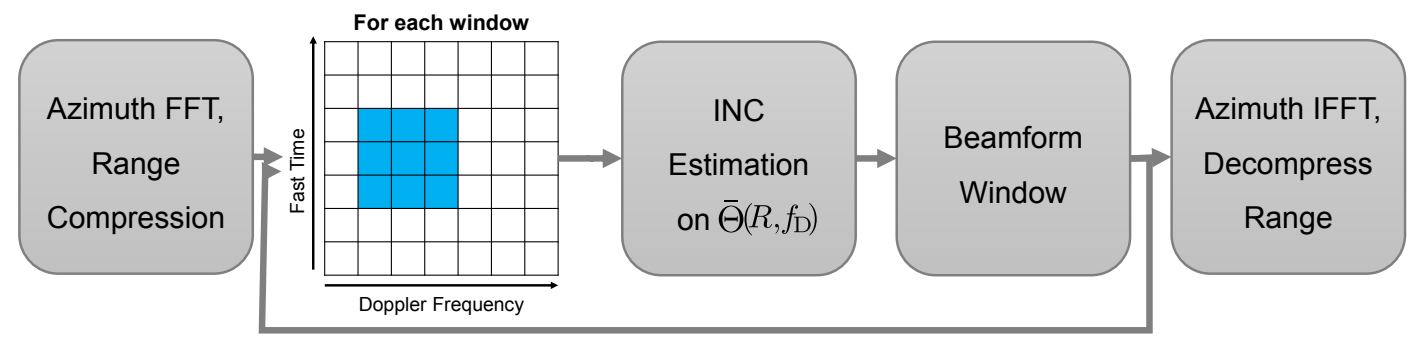

Figure 15. Two-dimensional MVDR RFI mitigation with DBF. A mitigation of $(N-1)(M-1)$ interferers per window is possible.

By performing the 2DMVDR on small azimuth chunks, the RFI variability due to the moving platform can be better compensated. Additionally, the impact of topography can be better handled. For a radar with a wide aperture (long integration time), the algorithm should be applied on multiple 
azimuth sublooks if the topography variation is large. An alternative would be an integration of the 2DMVDR into a backprojection focusing algorithm [49,50].

\subsection{Summary}

A summary of the presented algorithms is listed in Table 2. 
Table 2. Summary of the presented algorithms.

\begin{tabular}{ccccc}
\hline Algorithm & DBF Type & Placing of up to N-1 Nulls per & RFI Type & Advantage \\
\hline Pulse-Wise & Elevation & per pulse & Both & $\begin{array}{c}\text { Fast, for scenes without expected in-swath } \\
\text { interference }\end{array}$ \\
\hline Segment-Wise Frequency & Elevation & per frequency bin & Continuous & $\begin{array}{c}\text { Fast, for scenes without expected in-swath } \\
\text { interference }\end{array}$ \\
\hline Range-Dependent Time & Elevation & per range line & Continuous & $\begin{array}{c}\text { Recovers part of swath despite in-swath } \\
\text { interference }\end{array}$ \\
\hline Range-Dependent Frequency & Elevation & per range window and frequency bin & Continuous & $\begin{array}{c}\text { Recovers part of swath despite in-swath } \\
\text { interference. Beneficial for smaller antenna } \\
\text { arrays. }\end{array}$ \\
\hline Pulsed-RFI & Elevation & per range window & Pulsed & $\begin{array}{c}\text { Suitable for pulsed RFI Scenarios. Recovers } \\
\text { in-swath interference }\end{array}$ \\
\hline Doppler Dependent & Azimuth & per Doppler frequency bin & Continuous & $\begin{array}{c}\text { Fast, for scenes without expected in-swath } \\
\text { interference }\end{array}$ \\
\hline Doppler Dependent Frequency ting time & Azimuth & per range-Doppler window & Both & $\begin{array}{c}\text { Suitable for CW and pulsed in-swath } \\
\text { interference }\end{array}$ \\
\hline 2D & Both & per range-Doppler window & Both & $\begin{array}{c}\text { Capable of placing most notches and can } \\
\text { recover largest swath percentage }\end{array}$ \\
\hline
\end{tabular}




\section{Simulations for DBF in Elevation}

The previous sections introduced new RFI mitigation algorithms for SAR based on DBF. The goal of this section is to analyze and to compare the performance of the algorithms that utilize DBF in range, before they are applied to experimental data in Section 5. The RFI mitigation algorithms are applied without any knowledge about the simulated scene.

\subsection{Simulation Steps and Parameters}

As an initial step, multi-channel SAR raw data is simulated in baseband as if acquired by a planar antenna in an altitude of $3.2 \mathrm{~km}$. The antenna is aligned horizontally to be in agreement with the experimental results. However, the tilt of the antenna does not affect the results significantly. The channel spacing is half a wavelength. The channel number (number of array elements) $N$ ranges from 2 to 64 . Even though the total length of antennas are proportional to the number of elements, this is an inevitable design requirement for DBF antennas. Having antenna elements longer than half wavelength would increase grating lobes in the antenna pattern, significantly impacting the DBF performance.

It is therefore important to note that two effects are visible in the simulations. First, the increased number of $\mathrm{N}$ increases the number of notches that can be placed in the antenna pattern. The ability of the algorithms to handle a specific number of interferers can therefore be attributed to the increase of the number of antenna subelements $N$. On the other hand, the increased antenna length sharpens the main beam. It improves the angular resolution that separates the instantaneous SAR signal from the interferer signals in the angular domain. Therefore, the increased antenna length is responsible for the improvement of the total percentage of the swath that can be recovered with increasing antenna size.

The observed scene is simulated from an incidence angle of $21^{\circ}$ to $60^{\circ}$ and with a complex-Gaussian circular backscatter. The transmit chirp of the simulated system is $20 \mu$ s long and occupies a bandwidth of $120 \mathrm{MHz}$, sampled at $290 \mathrm{MHz}$. A total of 500 pulses are simulated at a center wavelength of $\lambda$. Note that the complex-Gaussian circular backscatter allows to simulate the 500 pulses without moving the platform (in contrary to the case of a non-Gaussian target). The SNRof the raw data is set to two values: (a) $0 \mathrm{~dB}$ to simulate the worst case of an extended target with identical SNR in the focused image and (b) $37.63 \mathrm{~dB}$ which is equivalent to the range gain of a point target for the simulated chirp. Next, an RFI signal is simulated that is received by the same multi-channel SAR system. The RFI type is a CW interferer (note that the RFI type is irrelevant to a spatial filter such as DBF). The exact RFI specifications (location and transmit frequency) are presented in the following subsection. All parameters are summarized in Table 1 . The data is then processed according to Figure 16. Both SAR and RFI data, are compressed in range (Note that this is only necessary in the airborne scenario to reduce the angular pulse extent. This is not necessary in the spaceborne case and could also be achieved with dispersive beams). SCORE beamforming is applied to the SAR data to produce the ideal reference data. The contaminated multi-channel data is synthesized by adding the range-compressed SAR and RFI data. The contaminated dataset is then processed with the previously presented RFI mitigation algorithms. Because the channels are aligned in elevation, the selected algorithms are the Pulse-Wise MVDR, RD-Time MVDR and RD-Frequency MVDR. The output of the algorithm is the recovered data. Finally, the recovered data is divided by the ideal reference data. The output is thus a residual error image that can be analyzed further according to the multiplicative error model presented in the next subsection. 


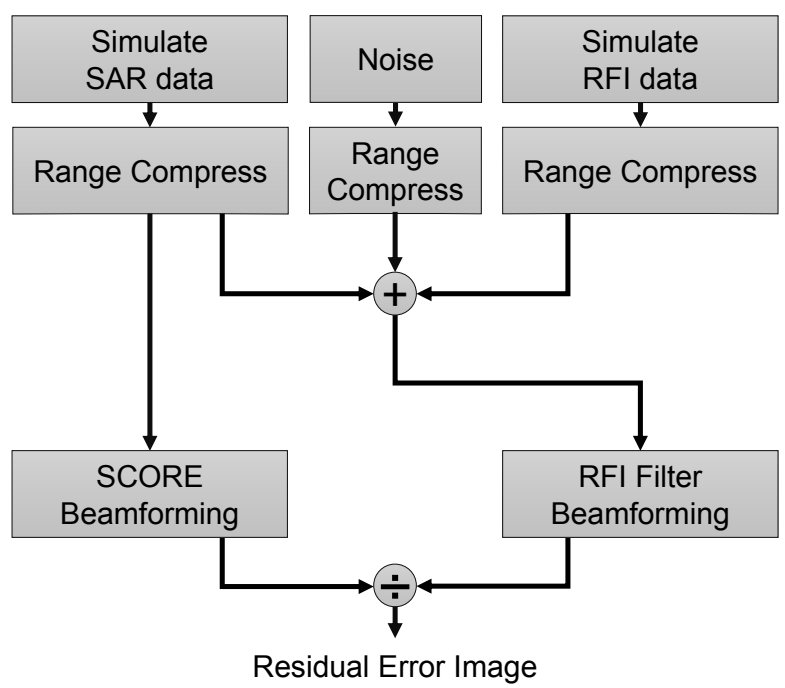

Figure 16. Simulation chain for the performance evaluation of the RFI mitigation. The simulated SAR and RFI data are range compressed individually. The range-compressed SAR data is then beamformed with SCORE to create the ideal reference data. Contaminated data is produced by summing the range-compressed SAR and RFI data. The contaminated data is then corrected using the algorithms. In the end, the algorithm output is divided by the reference data to produce the residual error image.

\subsection{Error Model}

Figure 17a illustrates the use of an additive error model because RFI is of an additive nature. The black vector represents the SAR signal vector. An additive error with fixed amplitude (blue-dashed vector) will displace the SAR signal vector to a location on the green-dashed circle. The exact position is determined by the relative phase of the interferer which can rotate the error vector along the red line. It is evident that the resulting gain error will depend on the interferer phase, which results in amplification or attenuation of the signal for constructive and deconstructive interference respectively. This mean that the additive amplitude error alone gives no indication of the resulting signal gain. To ensure that the radiometric budget is met, one can not set a fixed limit to the phase and amplitude error but only to the combination of both. Further, the resulting signal phase error will depend on the ratio of the RFI amplitude (blue-dashed) to the SAR amplitude (black) and the RFI phase (red). The larger the relation of the RFI amplitude is relative to the SAR amplitude, the greater the introduced phase error for a fixed RFI amplitude.

In the same manner, the additive phase error alone gives no indication of the resulting signal phase. Given these points, the interdependency of the signal error on the RFI amplitude, RFI phase and SAR amplitude makes the additive error model a disadvantageous choice. In contrast, a multiplicative error model is illustrated in Figure 17b. Note that a conversion between both models is straightforward. For this model, the multiplicative amplitude error describes the resulting gain (blue-dashed) and the multiplicative phase error describes the resulting signal phase (red). Both errors are decoupled and a fixed limit for both errors can be defined individually. For this reason, the error model used in this paper is multiplicative and given by

$$
s_{\mathrm{Err}}(\tau, t)=\left[A_{0}+A(\tau, t)\right] \cdot s_{\mathrm{R}, \mathrm{DBF}}(\tau, t) \cdot e^{j \Phi_{0}} \cdot e^{j \Phi(\tau, t)},
$$

where $A_{0}$ and $\Phi_{0}$ are constant amplitude and phase offsets and $A(\tau, t)$ and $\Phi(\tau, t)$ are time-varying errors. $s_{\mathrm{R}, \mathrm{DBF}}(\tau, t)$ is the beamformed $s_{\mathrm{R}}(\tau, t, m)$ signal. The four parameters are derived from the raw data because the simulated SAR signal is an extended target that does not undergo a processing gain. $A(\tau, t)$ and $\Phi(\tau, t)$ are expressed with their respective standard deviations $\sigma_{\mathrm{A}}$ and $\sigma_{\Phi}$. 


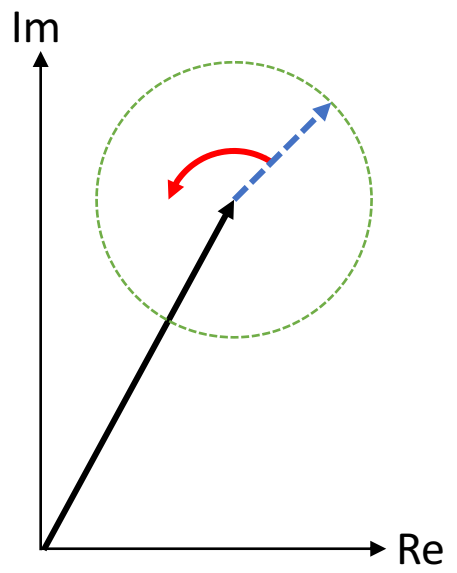

(a)

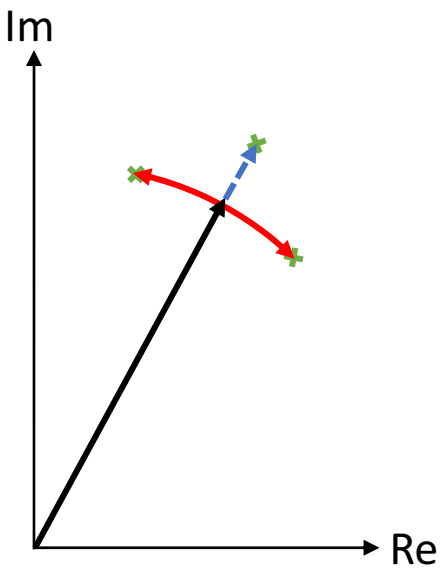

(b)

Figure 17. (a) Additive error model: SAR signal (black vector) imposed with additive RFI (blue-dashed vector). A phase change of the interferer (red) causes the measured signal to be within the green-dashed circle. The resulting gain error depends on both RFI amplitude and phase. The resulting phase error depends on amplitude and phase as well. (b) Multiplicative error model: SAR signal (black vector) that is affected by multiplicative gain error (blue-dashed vector) and multiplicative phase error (red). The resulting gain error depends on the multiplicative gain, the resulting phase error on the multiplicative phase.

\subsection{Simulated Interference Scenarios}

Four different interferer scenarios are considered for the performance analysis. The number of interferers is different in each case. This allows the testing of the algorithms under different conditions and to determine their filtering capabilities. In order to demonstrate the performance of various number of antenna elements, we keep the number of RFI sources the same as we increase the number of antenna elements. For cases where number of antenna elements go beyond the number of RFI sources, the methods show different performances which is not only contributed to the Capon AoA estimation performance but also to the antenna pattern notching capability that increases with $N$. In a real data collection, the RFI environment is not known to the radar operators in advance yet it is likely a combination of the scenarios that are described next.

Note that an out-of-swath interferer is seen through the sidelobes at all times. An in-swath interferer moves through the sidelobes, into the main beam and back into the sidelobes as the beam scans in a scan-on-receive fashion.

\subsubsection{Scenario A}

The first scenario consists of a single interfering source that is located outside of the swath at $-20^{\circ}$ and transmits at a baseband frequency of $40 \mathrm{MHz}$. A single interferer represents the easiest case and it is expected that all algorithms can filter the interferer regardless of the number of available channels. Because the interferer is outside of the swath, it is possible to fully recover the SAR data. This scenario is depicted in Figure 18.

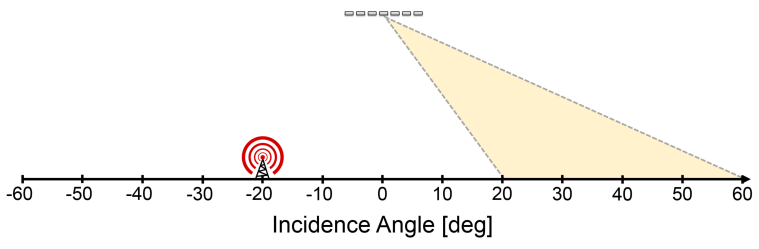

Figure 18. Scenario A: A single interferer is located outside the swath at $-20^{\circ}$. It is transmitting a signal at $40 \mathrm{MHz}$ in baseband. 


\subsubsection{Scenario B}

An additional interferer is placed inside the swath at $40^{\circ}$ for Scenario B (Figure 19). This interferer is transmitting at a baseband frequency of $25 \mathrm{MHz}$. Because it is located inside the swath, the SAR signal can not be recovered in its angular proximity. However, it is expected that the filters can recover part of the swath. The percentage of the swath that can be recovered will depend on the algorithm capabilities.

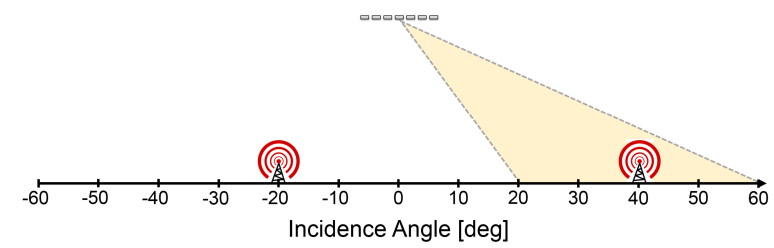

Figure 19. Scenario B: Two interferers are present. The first interferer is outside the swath at $-20^{\circ}$ with baseband frequency $40 \mathrm{MHz}$. The second interferer is inside the swath at $40^{\circ}$ with baseband frequency $25 \mathrm{MHz}$.

\subsubsection{Scenario C}

The number of interferers is increased to 11 for Scenario C (Figure 20). All interferers are located outside of the swath, which makes a full recovery of the SAR signal possible. The interferers are equally spaced in $5^{\circ}$ intervals starting from $-50^{\circ}$ to $0^{\circ}$. Each interferer is transmitting at a different frequency, starting from $-60 \mathrm{MHz}$ to $25 \mathrm{MHz}$ in baseband with a $5 \mathrm{MHz}$ spacing. The quality of the recovery is expected to depend on the number of channels $N$ and on the used algorithm.

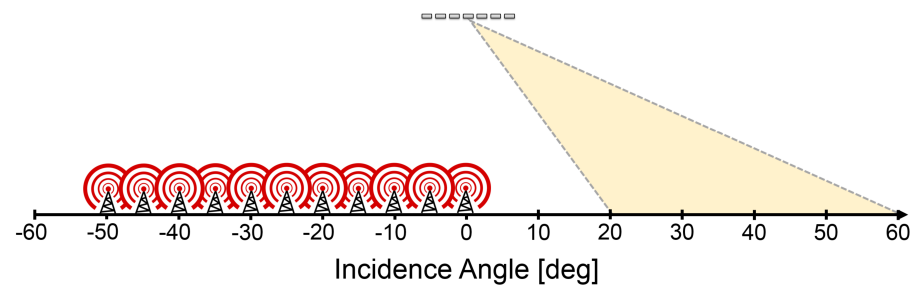

Figure 20. Scenario $C: 11$ interferers are present and outside of the swath. Locations range from $-50^{\circ}$ to $0^{\circ}$ in $5^{\circ}$ intervals. Transmitting frequencies range from $-60 \mathrm{MHz}$ to $25 \mathrm{MHz}$ in baseband with $5 \mathrm{MHz}$ spacing.

\subsubsection{Scenario D}

The number of interferers remains at 11 for Scenario D (Figure 21). However, the interferer spacing is changed and four interferers are moved inside of the swath, which prevents a full recovery of the SAR signal. The interferers are now equally spaced in $10^{\circ}$ intervals starting from $-50^{\circ}$ to $50^{\circ}$. The interferers transmitting frequencies stay the same (starting from $-60 \mathrm{MHz}$ to $25 \mathrm{MHz}$ in baseband with a $5 \mathrm{MHz}$ spacing). Reconstructing the SAR data is a challenge because the in-swath interferers are spread over the entire swath. The recovery is expected to improve with the number of channels. However, the RD-Frequency MVDR is expected to perform the best in this scenario because of its ability to change the notches adaptively in the frequency domain. 


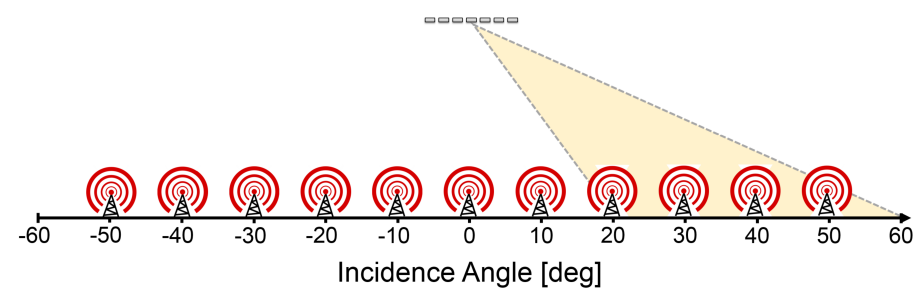

Figure 21. Scenario D: 11 interferers are present. They are located inside and outside of the swath. Locations range from $-50^{\circ}$ to $50^{\circ}$ in $10^{\circ}$ intervals. Transmitting frequencies range from $-60 \mathrm{MHz}$ to $25 \mathrm{MHz}$ in baseband with $5 \mathrm{MHz}$ spacing.

\subsection{Simulation Results}

An important aspect of the presented algorithms is the estimation of the INC matrix by integrating a spatial spectrum estimate. The spatial spectrum is estimated with the Capon method because of its robustness against false peaks in the spectrum and its low computational complexity. This choice comes at the expense of a worsening angular resolution $\delta \theta_{\mathrm{el}}$ with smaller SNR. In Figure 22 the spatial spectrum estimate for a range line of Scenario A is shown. Because the data is range-compressed, the instantaneous SAR signal return from the picked range line is coming from $54^{\circ}$. The RFI source is located at $-20^{\circ}$. Note that the spectrum estimation is performed at the center frequency of the radar. Because the interferer is shifted from the center frequency, this causes an angular offset $\Delta \theta_{\mathrm{RFI}}$ in the spatial estimate, as the phase ramp induced for $\theta_{\mathrm{RFI}}$ at the interferer frequency $f_{\mathrm{RFI}}$ is equivalent to a phase ramp of different AoA at $f_{\mathrm{c}}$ :

$$
\frac{2 \pi d f_{\mathrm{c}}}{\mathrm{c}_{0}} \sin \left(\theta_{\mathrm{RFI}}\right)=\frac{2 \pi d f_{\mathrm{RFI}}}{\mathrm{c}_{0}} \sin \left(\theta_{\mathrm{RFI}}+\Delta \theta_{\mathrm{RFI}}\right)
$$

This shift is acceptable because once the algorithm places a notch in the antenna pattern towards $\theta_{\mathrm{RFI}}+\Delta \theta_{\mathrm{RFI}}$ for $f_{\mathrm{c}}$, it is effectively placing a notch towards $\theta_{\mathrm{RFI}}$ for $f_{\mathrm{RFI}}$. It is also evident from Figure 22 that the two different SNRs yield different resolutions in the spatial spectrum. While the SNR of $37.63 \mathrm{~dB}$ shows a good ability to resolve both signals accurately and with low side lobes, the SNR of $0 \mathrm{~dB}$ results in wide peaks with high side lobes. Note that an improved resolution $\delta \theta_{\mathrm{el}}$ can be achieved with more complex spectrum estimators at the cost of computational load. In the following subsections it will be shown that the algorithms still perform reasonably good at SNR of $0 \mathrm{~dB}$. However, the resolution $\delta \theta_{\mathrm{el}}$ in the spatial spectrum impacts the ability to suppress interference in the swath as the widening peak increases the visible PE. For the SNR $=0 \mathrm{~dB}$ simulation, the integration gap around the PE is therefore choosen so that the INC is not integrated over the instantaneous SCORE main beam, as discussed before. By this means, a notch that is placed close to the instantaneous SAR signal because of the widened PE does not degrade the antenna pattern. For the SNR $=37.63 \mathrm{~dB}$ simulation, this integration gap is reduced to a quarter of the main beam width because the signals are better resolved in the spatial spectrum and demonstrate what is possible with more complex spatial estimators [45-47]. 


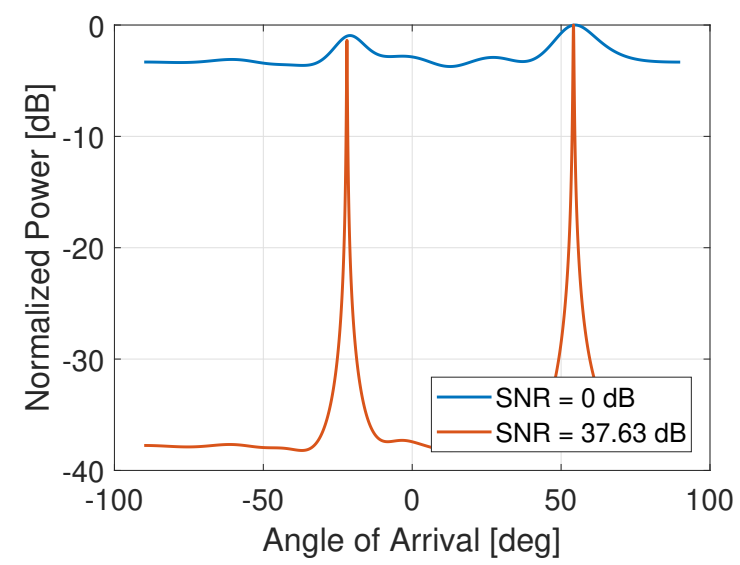

Figure 22. Capon spatial spectrum estimate of range-compressed range line with instantaneous SAR signal coming from $54^{\circ}$ and RFI source at $-20^{\circ}$.

\subsubsection{Scenario A}

Scenario A consists of a single interferer outside of the swath. For this reason, a spatial filter seems to be ideal to suppress the RFI and a full recovery of the swath is expected. Figure 23 shows the phase standard deviation of the residual error image (as defined in Section 4.2) measured in each range line (and thus at different incidence angles) before and after spatial filtering with the RD-Frequency MVDR beamformer ( $\mathrm{SNR}=37.63 \mathrm{~dB}$ ). The RFI-Noise-Ratio (RNR) is varied along the $y$-axis from $-20 \mathrm{~dB}$ to $40 \mathrm{~dB}$ and represents the power at each subelement. It is expected that the more elements $N$ the antenna array has, the stronger the RFI powers it can suppress because more notches are available. Figure 23a,c show the error before the spatial filter is applied for $N=8$ and $N=32$, respectively. The first observation is that the degradation of the data begins earlier for $N=8$ at a RNR of $-15 \mathrm{~dB}$. The reason for this is that the longer array $(N=32)$ achieves a higher gain towards the swath and thus has a higher RFI suppression that is inherent to the antenna pattern. The data starts to degrade for $N=32$ at a RNR of $-5 \mathrm{~dB}$. The ripples in the error image are caused by the SCORE operation that results in the interferer passing through the nulls and peaks of the side lobes. The residual phase standard deviation error after applying the RD-Frequency MVDR beamformer is shown in Figure 23b $(N=8)$ and Figure 23d $(N=32)$. It is evident that the residual error drops back to the noise floor for both cases.

The mean $\mu_{\sigma_{\Phi}}$ and variance $\sigma_{\sigma_{\Phi}}^{2}$ of the phase standard deviation error $\sigma_{\Phi}$ is computed and the 3 sigma phase standard deviation error is defined as

$$
\mu_{\sigma_{\Phi}}+3 \sigma_{\sigma_{\Phi}}
$$

The difference between the ideal noise floor and the residual after filtering RFI is plotted as the 3 sigma phase standard deviation error increase in Figure 24. Figure 24a through Figure 24d show the results for $N=2,4,8,16,32$ and 64 , respectively. The solid colored lines represent the different algorithms for $\mathrm{SNR}=0 \mathrm{~dB}$ and the dashed colored lines represent them for $\mathrm{SNR}=37.63 \mathrm{~dB}$.

The black lines show the 3 sigma increase before the algorithms are applied (note that the black line performs best in the absence of RFI because the algorithms introduce a small processing noise). They are showing low values for low RNR and suddenly increase when the RFI starts to degrade the data. From Figure 24a through Figure 24d it is evident that the sudden data degradation occurs at higher RNR values with increasing antenna array length because of the aforementioned reason. The results of the Pulse-Wise MVDR algorithm are shown in blue. Starting from $N=8$, the 3 sigma increase is below $2.5^{\circ}$ regardless of SNR and RNR. For lower $N$, the Pulse-Wise MVDR is not behaving stably. As $N$ increases, the maximum 3 sigma increase drops. For $N=16$ the increase is below $1^{\circ}$ and for higher $N$ it is only a fraction of a degree. The RD-Time MVDR is shown in orange and shows a 
stable behavior even for $N=2$. However, at $N=2$ it is only able to suppress the RFI up to a RNR of $0 \mathrm{~dB}$ for $\mathrm{SNR}=0 \mathrm{~dB}$ (up to a RNR of $5 \mathrm{~dB}$ for $\mathrm{SNR}=37.63 \mathrm{~dB}$ ). This is because only one narrow notch can be placed which makes the algorithm sensitive to a slight misplacement of the notch. For larger $N$, a wider notch is placed that suppresses the RFI even if a slight misplacment occurs. For $N=4$, the 3 sigma increase of the RD-Time MVDR is constantly below $3^{\circ}$ and for $N=8$ it is constantly below $1.5^{\circ}$. After $N$ exceeds 16, the algorithm reaches its best performance with a 3 sigma increase below $0.43^{\circ}$. A further increase of $N$ does not improve the algorithm anymore. In addition, the constant 3 sigma increase causes a degradation of the data by these $0.43^{\circ}$ compared to the uncorrected case for low RNR. The RD-Frequency MVDR beamformer (green) shows a similar behavior to the RD-Time MVDR. The maximum error shown by the green curves are below the orange curves for $N=2$ to 8 . Hence, the RD-Frequency MVDR beamformer shows a better performance than the RD-Time MVDR because the available notches can be distributed differently at different frequencies. This changes for larger $N$ and the maximum and constant 3 sigma increase at $N=16$ is 0.62 . This can be explained by the fact that the RD-Frequency MVDR injects a higher artifical noise floor than the RD-Time MVDR. This is likely due to the additional processing with windows in the frequency domain. This artificial noise floor rises close to $2^{\circ}$ for $N=32$ to 64 . Overall, all presented RFI suppression algorithms based on DBF are able to handle Scenario A, which consist of a single interferer outside of the swath. However, the Pulse-Wise MVDR shows an inferior behavior for $N$ below 16 but achieves a better suppression if a lot of notches are available (and only out-of-swath interference is present). The RD-Time MVDR and RD-Frequency MVDR inject artificial noise that can degrade the data in the absence of RFI.

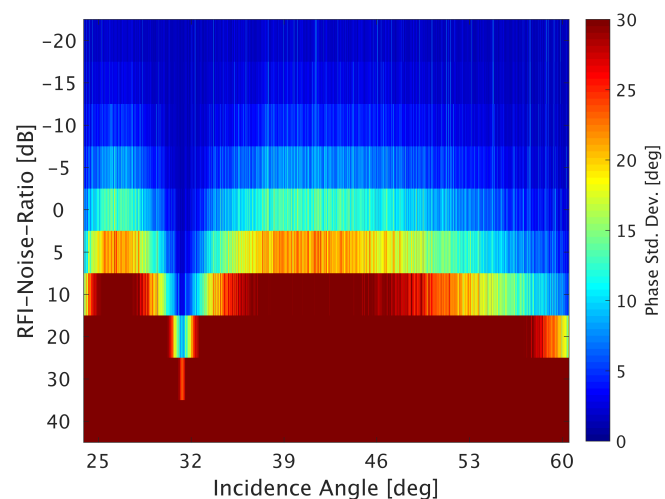

(a)

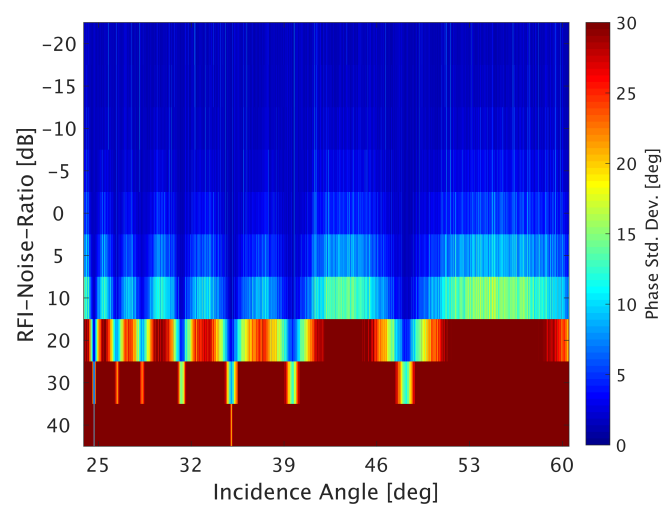

(c)

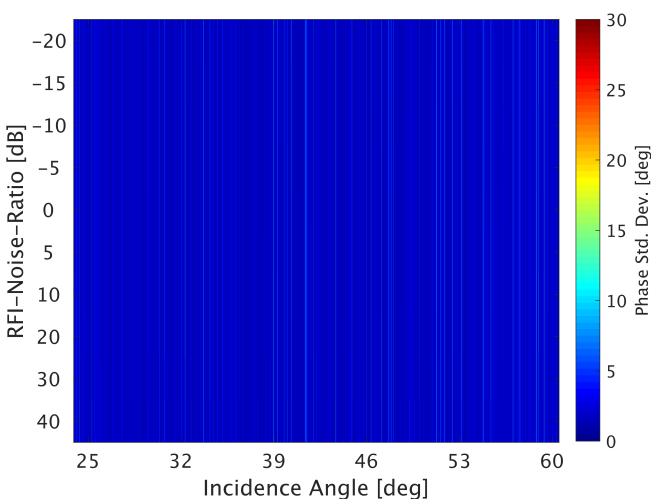

(b)

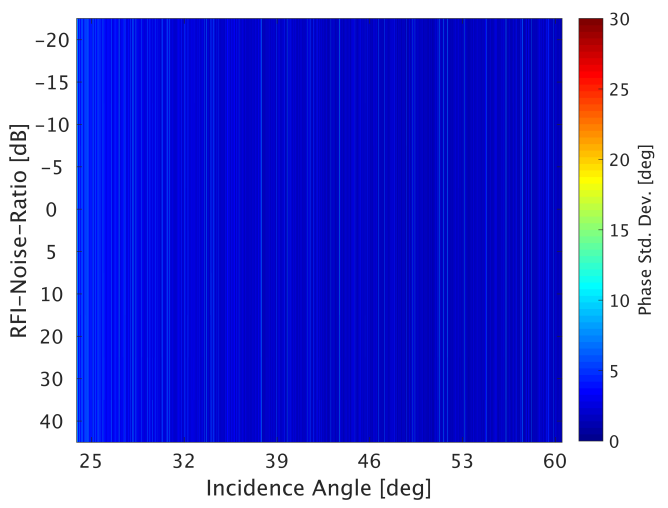

(d)

Figure 23. The phase standard deviation of the residual error image of scenario A for different RFI-Noise-Ratios versus the incidence angle of the instantaneous SAR signal. The SNR is $37.36 \mathrm{~dB}$. (a) No RFI mitigation for $N=8$, (b) After RD-Frequency MVDR beamforming for $N=8$, (c) No RFI mitigation for $N=32$, (d) After RD-Frequency MVDR beamforming for $N=32$. 
The maximum 3 sigma phase offset increase at $R N R=40 \mathrm{~dB}$ is plotted in Figure 25a in dependency of array length $N$. Due to the scaling of the y-axis, the black line for the error pre-filtering is not visible in the plot. All algorithms show an improvement up to $N=8$, which resembles the minimum array length to suppress the interferer at the given RNR. Again, the Pulse-Wise MVDR improves with increasing length while the RD-Time MVDR and RD-Frequency MVDR inject an artifical noise floor that is increasing slightly with $N$. Note that the maximum 3 sigma phase offset increase of this artificial noise floor is $0.8^{\circ}$.

The maximum 3 sigma amplitude offset increase at $R N R=40 \mathrm{~dB}$ is plotted in Figure $25 \mathrm{~b}$. This error directly affects the radiometric budget. For $N$ below 8 the suppression is not sufficient and a high gain bias is introduced. This is due to the RNR of $40 \mathrm{~dB}$. For $N=8$, the RD-Frequency MVDR shows the best suppression with a gain bias of $0.2 \mathrm{~dB}$ followed by the RD-Time MVDR with a gain bias of $0.3 \mathrm{~dB}$. The worst suppression is reached by the Pulse-Wise MVDR with a gain suppression of $0.53 \mathrm{~dB}$. The error drops further for all algorithms with increasing $N$. It is evident that the Pulse-Wise MVDR is capable of removing the gain bias for $N$ larger than 32 to a residual of $0.05 \mathrm{~dB}$. The RD-Time MVDR and RD-Frequency MVDR have a remaining gain bias of $0.12 \mathrm{~dB}$ and $0.09 \mathrm{~dB}$, respectively.

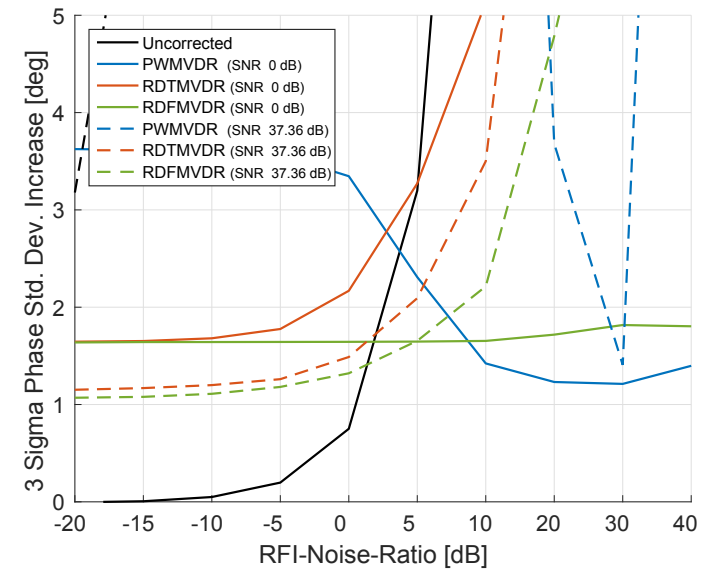

(a)

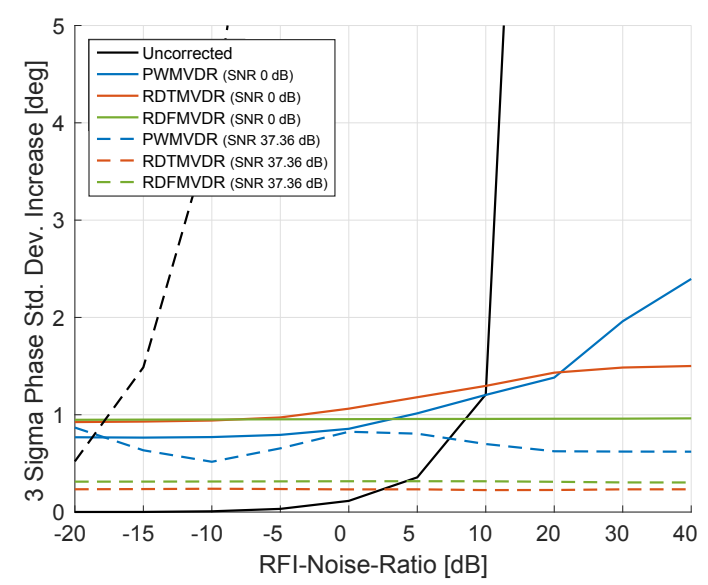

(c)

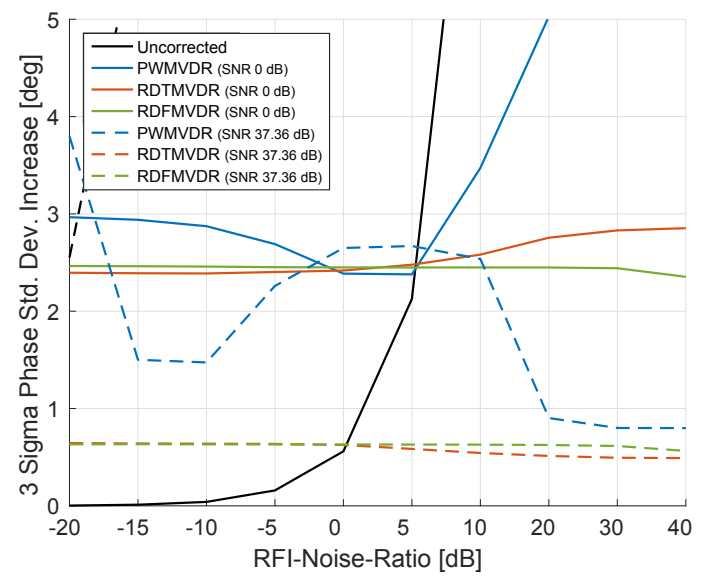

(b)

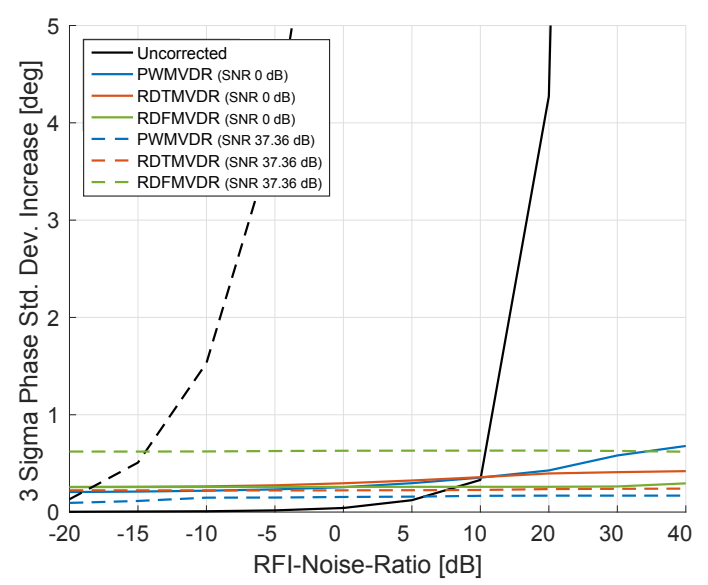

(d)

Figure 24. The 3 sigma phase standard deviation error of the residual error image of Scenario A versus RFI-Noise-Ratio. The plots are shown for $\mathrm{SNR}=0 \mathrm{~dB}$ (solid lines) and SNR $=37.36 \mathrm{~dB}$ (dashed lines). (a) $N=2$, (b) $N=4$, (c) $N=8$, (d) $N=16$. 


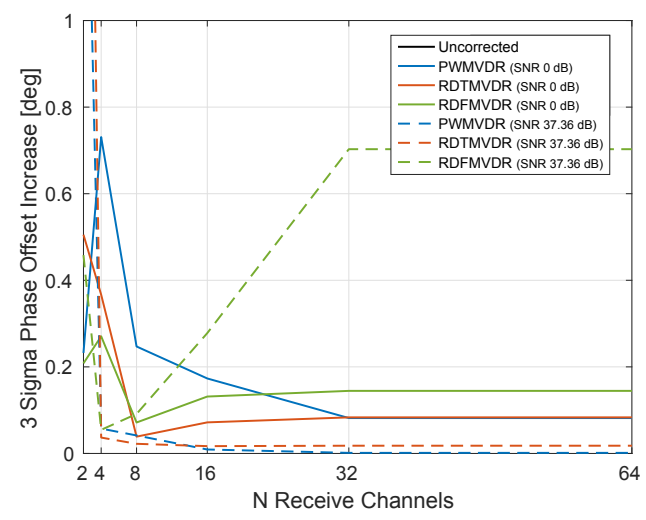

(a)

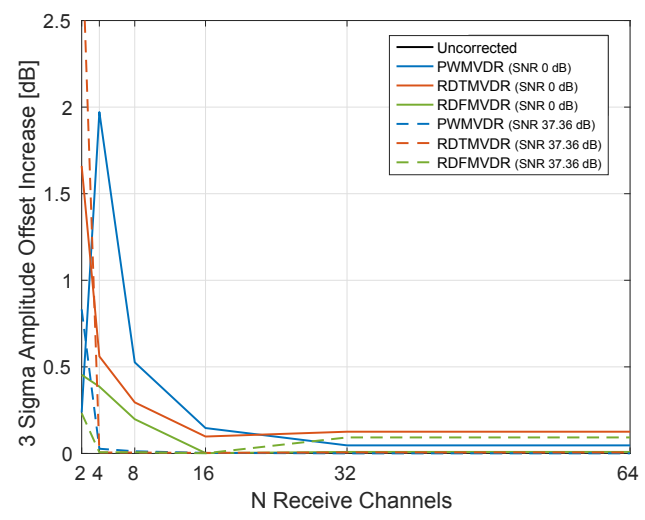

(b)

Figure 25. The 3 sigma error increase of the residual error image of scenario A at a RFI-Noise-Ratio of $40 \mathrm{~dB}$ vs. the antenna array length. The plots are shown for SNR $=0 \mathrm{~dB}$ (solid lines) and $\mathrm{SNR}=37.36 \mathrm{~dB}$ (dashed lines). (a) Phase offset, (b) Gain offset.

\subsubsection{Scenario B}

For scenario B, an additional interferer is put inside the swath at $40^{\circ}$. Because DBF is a spatial filter, part of the swath can not be recovered. The extent of the lost swath depends on the interferer power, the available notches (thus $N$ ), the beamwidth (thus antenna length) and the applied algorithm. Figure 26 shows the phase standard deviation of the residual error image measured in each range line before and after the RD-Frequency MVDR is applied ( $\mathrm{SNR}=37.63 \mathrm{~dB}$ ). Again, the RNR is varied along the $y$-axis from $-20 \mathrm{~dB}$ to $40 \mathrm{~dB}$ and represents the power at each subelement. Figure 26a,c show the error before the spatial filter is applied for $N=8$ and $N=32$, respectively. The first observation is that the degradation of the data sets for both figures already started at $-20 \mathrm{~dB}$. The degradation is more significant than for scenario A because the interferer inside the swath is not suppressed by the side lobes. However, the entire swath is lost sooner for $N=8$ than for $N=32$ due to the narrowing antenna pattern of the longer antenna. This results in a partial interference suppression with the SCORE operation when theinstantaneous main beam is not looking into the interferer. The residual phase standard deviation error applying the RD-Frequency MVDR beamformer is shown in Figure 26b $(N=8)$ and Figure 26d $(N=32)$. The residual error drops back to the noise floor in both cases up to a RNR of about $5 \mathrm{~dB}$. For stronger RNR, the interferer is causing a local degradation in the proximity of the interferer position. As expected, the width of the lost area around the interferer decreases with increasing $N$ (which here results in a longer antenna and thus a smaller instantaneous main beam). This is a fundamental limitation of DBF that can not be overcome by shaping the antenna pattern alone. Note that the remainder of the swath is recovered with the RFI suppression.

The amount of the lost swath is further analyzed in Figure 27. Figure 27a shows the phase standard deviation error at RNR $40 \mathrm{~dB}$ for each range line after the mean phase standard deviation error of the noise floor is subtracted $(N=16)$. The black lines show the error if no correction is performed. Note that the lower curve is due to the SNR of $0 \mathrm{~dB}$, whereas the higher black curve represents a SNR of $37.63 \mathrm{~dB}$. The blue lines show the error after the Pulse-Wise MVDR is applied. The more saturated blue shows the higher SNR. For both SNR, the Pulse-Wise MVDR is not effective in restoring part of the swath. This is expected because the Pulse-Wise MVDR is blind to the in-swath interferer due to the PE. As a consequence, the antenna pattern is the regular Scan-On-Receive pattern. As the radar scans in elevation to look at different incidence angles, the fixed interferer is wandering through the side lobes and periodic drops occur when the interferer is in an antenna pattern null. The orange lines show the result of the RD-Time MVDR beamformer. Part of the swath in the proximity of the interferer is lost but the remaining part of the swath is restored Note that, though the interferer is located at $40^{\circ}$, the antenna pattern sees it at a shifted angle due to the frequency offset from the center frequency (discussed in Section 2.4). The more saturated orange line for the better SNR case shows a smaller loss 
of the swath. The higher SNR can resolve the interferer position and the instantaneous SAR signal better because of the resolution in the Capon spectral estimate. The notch can therefore be placed closer to the instantaneous SAR AoA. The same behavior can be noticed for the RD-Frequency MVDR beamformer in green. However, the RD-Frequency MVDR is superior to the RD-Time MVDR because more of the swath can be recovered. The reason for this is that the RD-Frequency MVDR can place the notches more effectively due to the additional processing in the frequency domain. It can place different notches for the in-swath and out-of-swath interferer. The same plot is shown for $N=64$ in Figure 27b. The difference between this plot and the plot for $N=32$ is that more of the swath is recovered because the main beam width is getting smaller. In addition, the resolution of the Capon spectral estimate improves with $N$ and thus the resolution is improved for low SNR too.

The percentage of the recovered swath in dependency of $N$ is plotted from Figure 27c through Figure 27e for the phase standard deviation, the phase offset and the amplitude offset, respectively. The recovered swath in the figures has a phase standard deviation error below $20^{\circ}$, a phase offset below $5^{\circ}$ and an amplitude offset below $0.5 \mathrm{~dB}$. It is evident that the phase offset is the least sensitive parameter and the gain is the most sensitive parameter. For all parameters, some amount of swath can be recovered for $N$ larger than 4 . The lost swath halfens for a doubling of the anntenna length, which is due to the resulting halfening of the antenna pattern main beam. As a consequence, the improvement with $N$ up to $N=16$ is large and then starts to get smaller. In addition, it is evident that it is necessary to use the range-dependent algorithms to recover the swath. The RD-Frequency MVDR is especially superior for a smaller $N$. With increasing $N$, when a multiple of notches is available per interferer, the higher computational load can not be justified anymore and the benefit over the RD-Time MVDR diminishes.

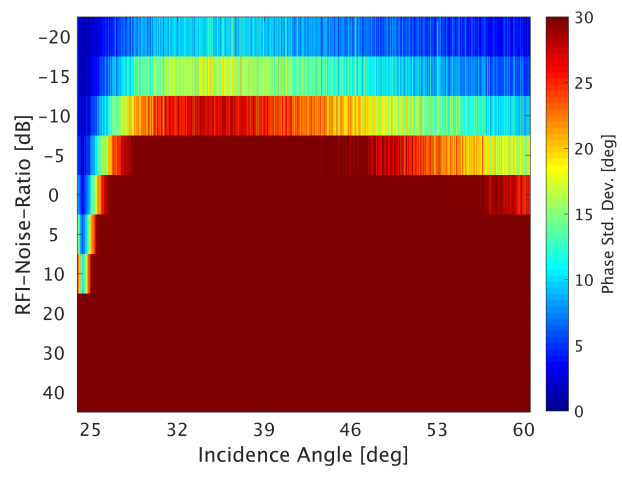

(a)

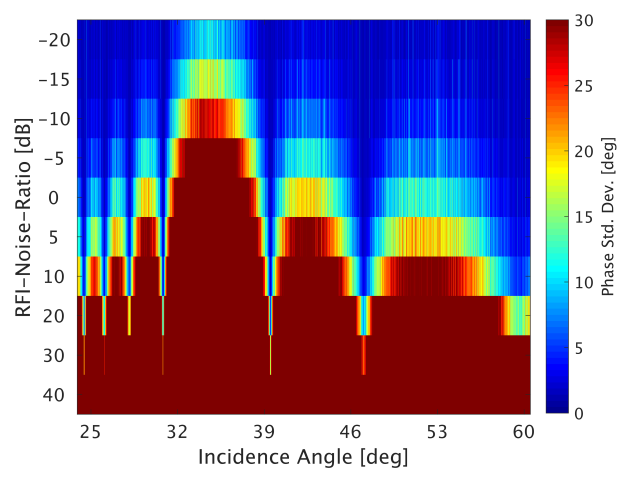

(c)

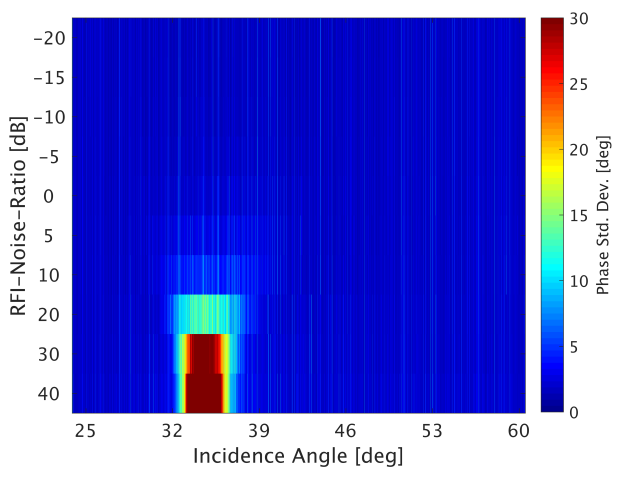

(b)

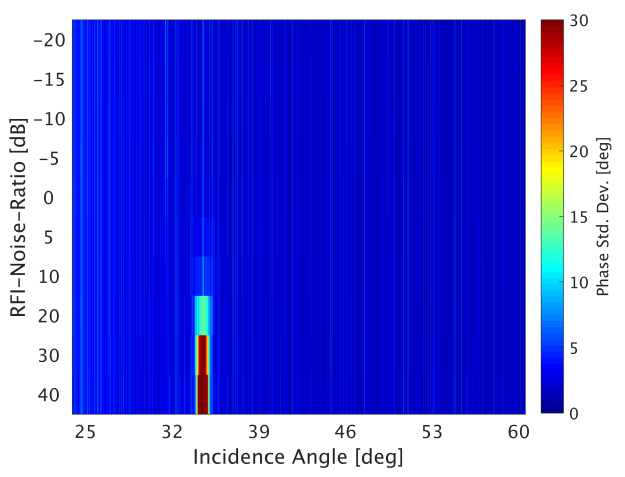

(d)

Figure 26. The phase standard deviation of the residual error image of scenario $B$ for different RFI-Noise-Ratios versus the incidence angle of the instantaneous SAR signal. The SNR is $37.36 \mathrm{~dB}$. (a) No RFI mitigation for $N=8$, (b) After RD-Frequency MVDR beamforming for $N=8$, (c) No RFI mitigation for $N=32$, (d) After RD-Frequency MVDR beamforming for $N=32$. 


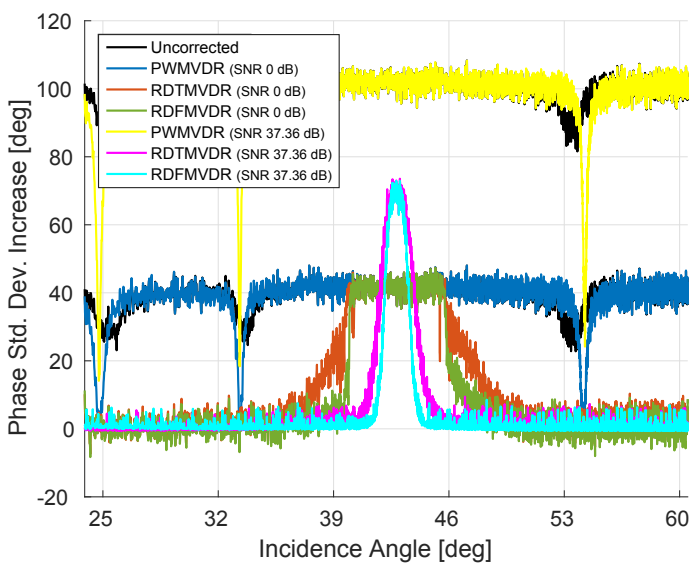

(a)

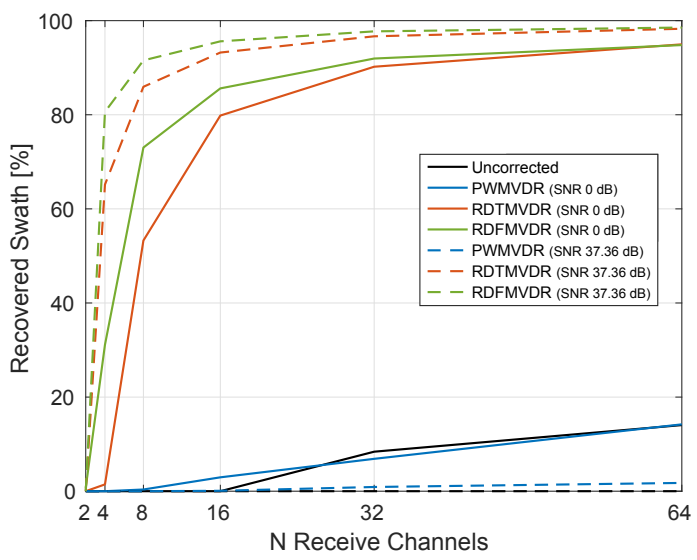

(c)

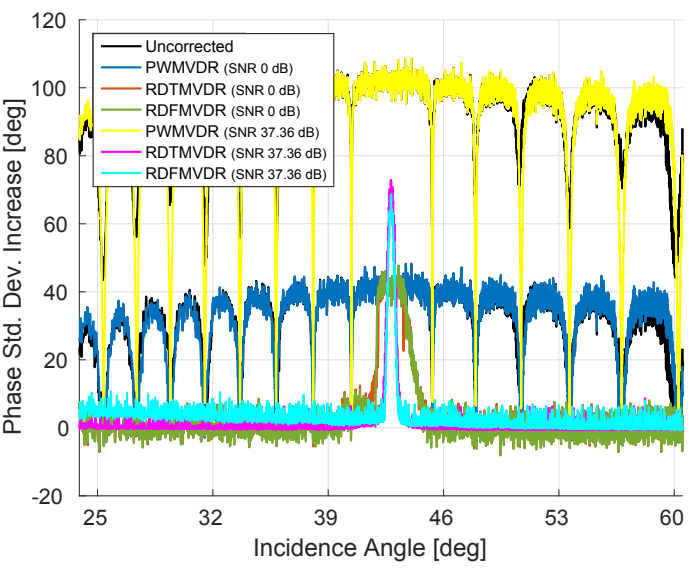

(b)

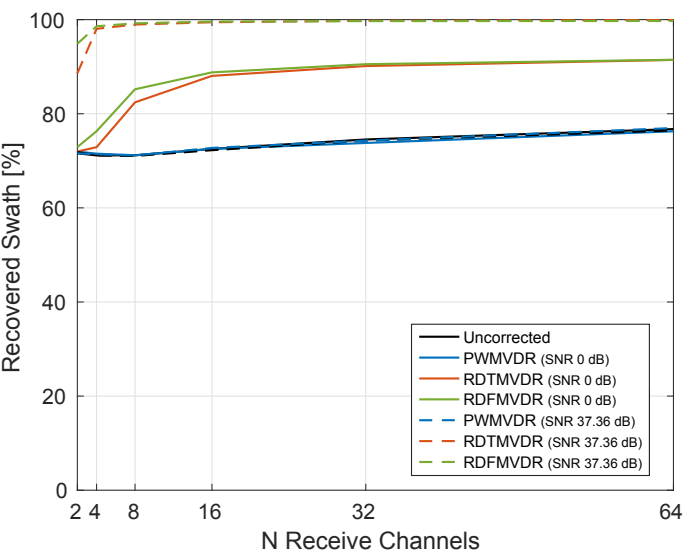

(d)

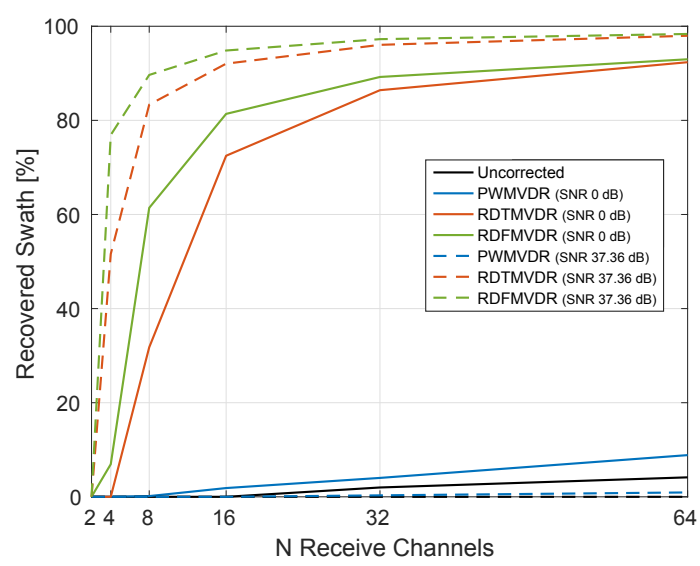

(e)

Figure 27. The phase standard deviation increase of the residual error image for scenario $B$ at a RFI-Noise-Ratio of $40 \mathrm{~dB}$. (a) Error plotted versus incidence angle for $N=16$ and for $\mathrm{SNR}=0 \mathrm{~dB}$ (pale lines) and $\mathrm{SNR}=37.36 \mathrm{~dB}$ (saturated lines), (b) Error plotted versus incidence angle for $N=64$ and for $\mathrm{SNR}=0 \mathrm{~dB}$ (pale lines) and SNR $=37.36 \mathrm{~dB}$ (saturated lines), (c) The percentage of the recovered SAR swath that is within the phase std. dev. limits of $20^{\circ}$, (d) The percentage of the recovered SAR swath that is within the phase offset limits of $5^{\circ}$, (e) The percentage of the recovered SAR swath that is within the absolute gain limits of $0.5 \mathrm{~dB}$. 


\subsubsection{Scenario C}

As before, Figure 28 shows the phase standard deviation of the residual error image measured in each range line before and after spatial filtering with the RD-Frequency MVDR beamformer $(\mathrm{SNR}=37.63 \mathrm{~dB})$. Figure 28a,c show the error before the spatial filter is applied for $N=8$ and $N=32$, respectively. All the interferers in scenario $C$ are placed outside the swath and an initial suppression by the antenna pattern side lobes occurs. Therefore, the data degradation does not start until RNR $=-15 \mathrm{~dB}$ and $\mathrm{RNR}=-5 \mathrm{~dB}$. Even though there are 11 interferers and Figure $28 \mathrm{~b}$ shows the spatial filter for $N=8$, the full swath can be recovered. Fewer notches than interferers are needed if all interferers are outside the swath, as is the case here, because a wide notch can suppress multiple close interferers. Hence, only a few notches are sufficient to improve the side lobe suppression over a wide span of angles. However, even higher RFI powers could be suppressed if $N$ were increased. As expected, increasing $N$ to 32 in Figure $28 \mathrm{~d}$ results in a full recovery and is only shown for completeness.

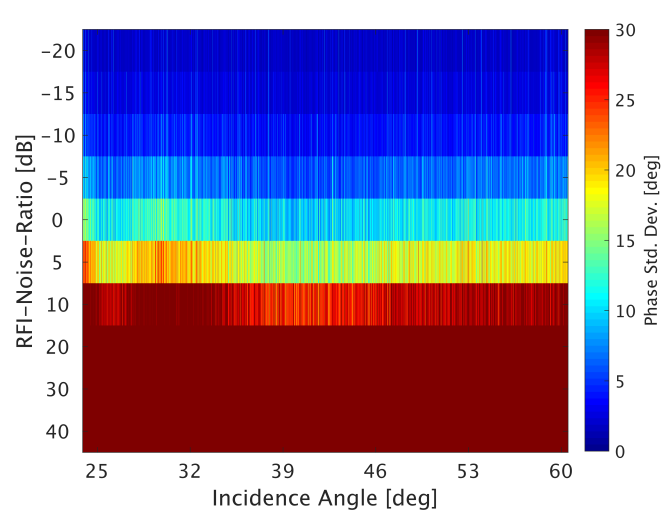

(a)

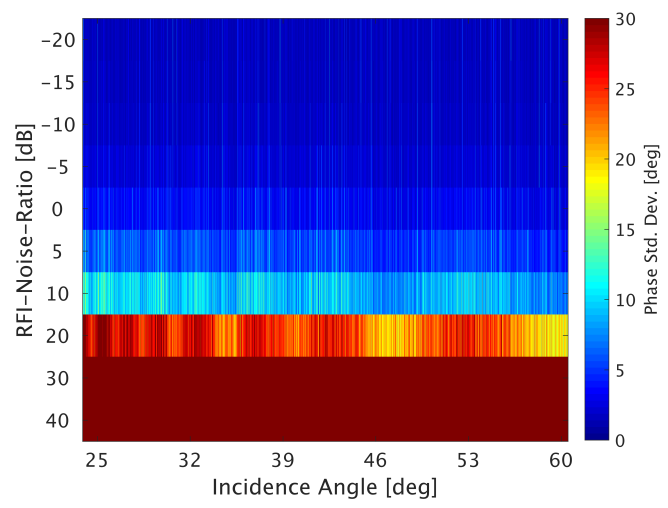

(c)

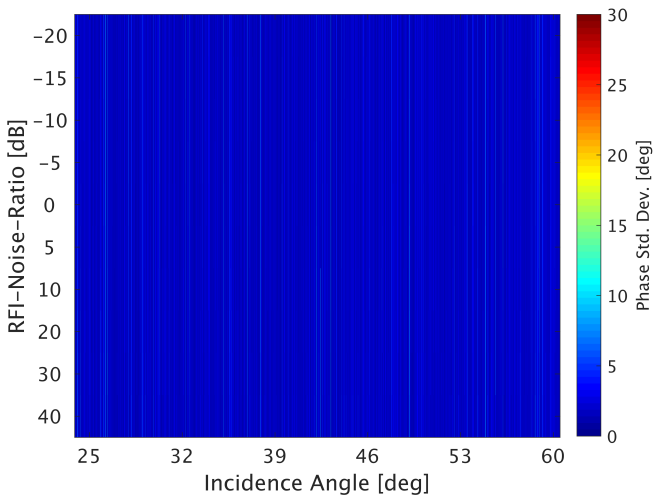

(b)

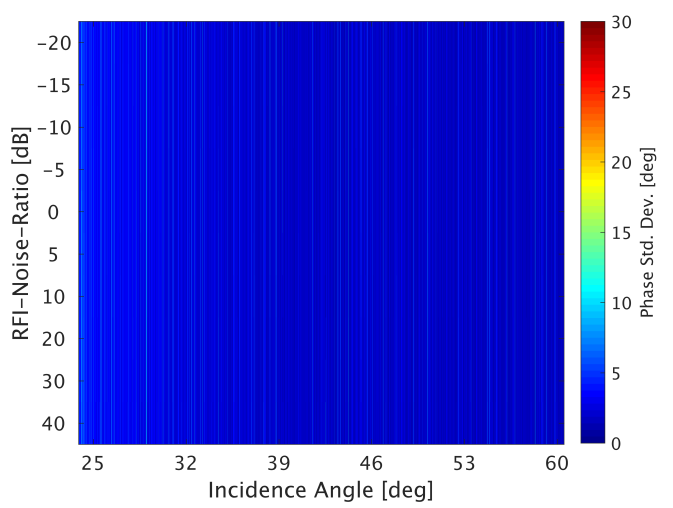

(d)

Figure 28. The phase standard deviation of the residual error image of scenario $C$ for different RFI-Noise-Ratios versus the incidence angle of the instantaneous SAR signal. The SNR is $37.36 \mathrm{~dB}$. (a) No RFI mitigation for $N=8$, (b) After RD-Frequency MVDR beamforming for $N=8$, (c) No RFI mitigation for $N=32$, (d) After RD-Frequency MVDR beamforming for $N=32$.

Next, Figure 29 shows the 3 sigma phase standard deviation error for scenario C. As before, $N$ increases from 2 to 64 and the beginning of the degradation (black line) is pushed to higher values. In the same manner, the colored curves after the spatial filtering are pushed to the lower left corner as $N$ increases. Because of the amount of interferers, the filtering with few notches and thus for low $N$ is degrading for high RNR. As in scenario A (single interferer) it can be observed that the fastest algorithm, the Pulse-Wise MVDR, performs the worst and the most complex algorithm, the RD-Frequency MVDR, 
the best. The disadvantage of the RD-Frequency MVDR is, besides the high computational load, an artifical noise floor that is injected and that becomes visible for large $N$. This artificial noise is on the order of $1.5^{\circ}$.

The maximum 3 sigma phase offset increase at $\mathrm{RNR}=40 \mathrm{~dB}$ is plotted in Figure 30a. The range-dependent algorithms show a phase offset of less than a degree above $N=8$. For high $N$, the limit of the phase offset reconstruction is $0.15^{\circ}$ for the RD-Time MVDR and $0.7^{\circ}$ for the RD-Frequency MVDR. The maximum 3 sigma amplitude offset increase at RNR $=40 \mathrm{~dB}$ is plotted in Figure 30b. Again, this error directly affects the radiometric budget. For $N$ below 8 the suppression is not sufficient and a high gain bias is introduced. This is due to the RNR of $40 \mathrm{~dB}$. For $N=8$, the RD-Frequency MVDR shows the best suppression with a gain bias of $1.25 \mathrm{~dB}$. The error drops further for all algorithms with increasing $N$. It is evident that the Pulse-Wise MVDR is capable of removing the gain bias for $N$ larger than 32. On the contrary, the RD-Time MVDR has a remaining gain bias of $0.09 \mathrm{~dB}$ and the RD-Frequency MVDR a remaining gain bias of $0.2 \mathrm{~dB}$. While the range-dependent algorithms show a better behavior for lower $N$, for large $N$ the gain bias of the Pulse-Wise MVDR is the best (if no interferer is inside the swath).

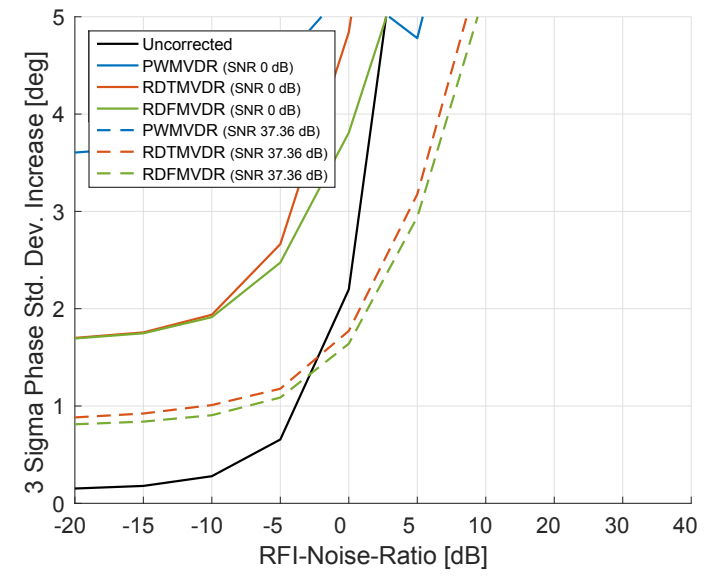

(a)

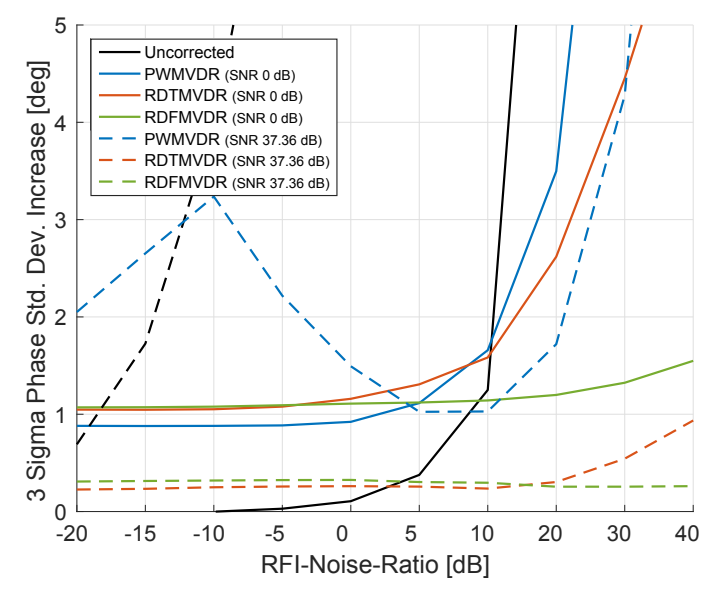

(c)

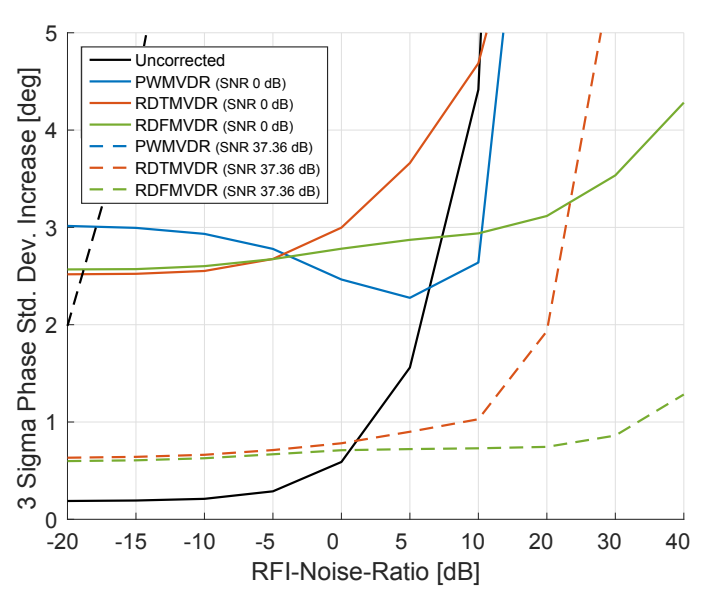

(b)

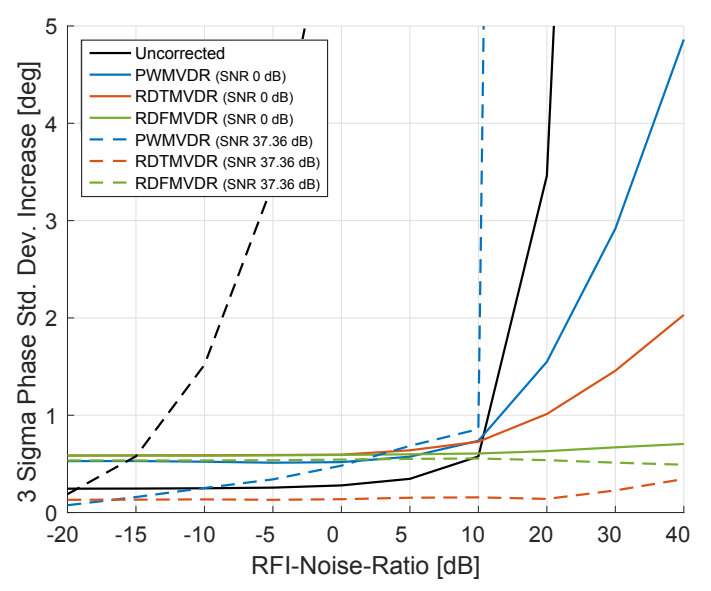

(d)

Figure 29. The 3 sigma phase standard deviation error of the residual error image of scenario $C$ versus RFI-Noise-Ratio. The plots are shown for SNR $=0 \mathrm{~dB}$ (solid lines) and SNR $=37.36 \mathrm{~dB}$ (dashed lines). (a) $N=2,($ b) $N=4$, (c) $N=8,($ d) $N=16$. 


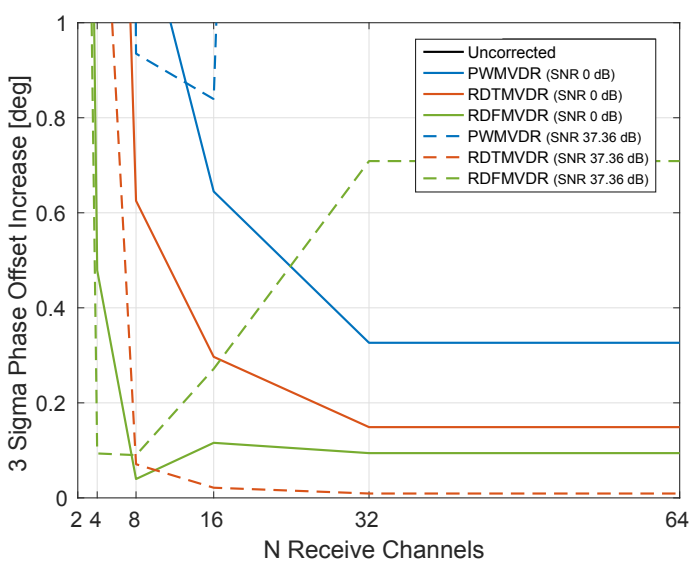

(a)

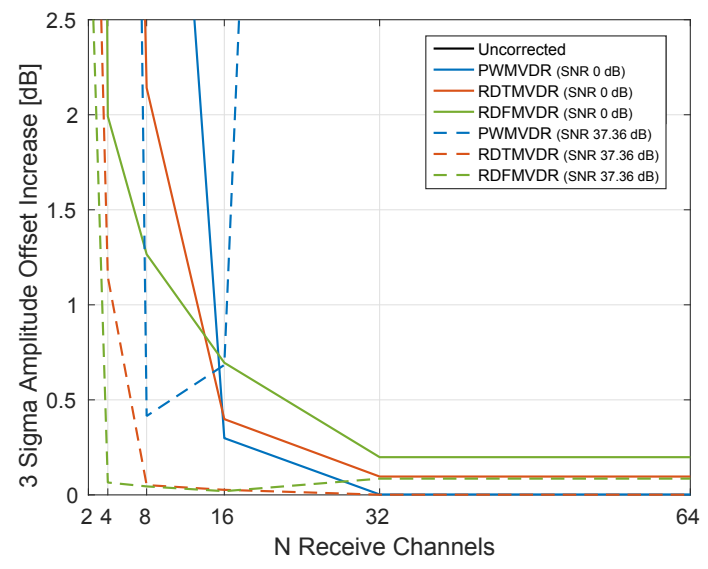

(b)

Figure 30. The 3 sigma error increaser of the residual error image of Scenario $C$ at a RFI-Noise-Ratio of $40 \mathrm{~dB}$ vs. the antenna array length. The plots are shown for SNR $=0 \mathrm{~dB}$ (solid lines) and $\mathrm{SNR}=37.36 \mathrm{~dB}$ (dashed lines). (a) Phase offset, (b) Amplitude offset.

\subsubsection{Scenario D}

In scenario D, 4 interferers are located inside the swath and 7 interferers outside of the swath. Again, it is expected that only part of the swath can not be recovered due to the in-swath RFI. Figure 31 shows the phase standard deviation of the residual error image measured in each range line (SNR $=37.63 \mathrm{~dB}$ ). Figure 31a,c show the error before the spatial filter is applied for $N=8$ and $N=32$, respectively. For $N=8$ all simulated RNR show a degradation of the entire swath as the main beam is constantly occupied by one interferer. The more narrow antenna pattern of $N=32$ (due to the longer antenna) shows the SCORE-typical ripples because the interferers move in and out of the main beam. Again, the increased antenna length (narrower instantaneous SCORE beam) yields an inherent suppression of the in-swath RFI to some extent. The residual phase standard deviation error applying the RD-Frequency MVDR beamformer is shown in Figure $31 \mathrm{~b}(N=8)$ and Figure $31 \mathrm{~d}$ $(N=32)$. The entire swath can be recovered in both cases for a RNR of up to $10 \mathrm{~dB}$. For this value, the interference is about $27 \mathrm{~dB}$ lower than the SAR signal. As the RNR values increase, only part of the swath can be recovered. As before, the higher $N$ (which here results in a longer antenna) improves the reconstruction area because of the more narrow antenna pattern. For $N=8$ the areas overlap and more than half of the first part of the swath is lost. Even though 4 interferers are placed in the swath, only 3 are visible. This can be explained with the fact that the frequency shift of one of the interferers results in an AoA shift at $f_{c}$, which moves the interferer outside of the swath from the perspective of the radar's center frequency (as discussed in Section 2.4).

The amount of the lost swath is further analyzed in Figure 32. As before, Figure 32a $(N=16)$ and Figure 32a $(N=64)$ show the phase standard deviation error at RNR $40 \mathrm{~dB}$ for each range line after the mean phase standard deviation error of the noise floor is subtracted. The Pulse-Wise MVDR (blue) curve is comparable to the uncorrected (black) curve. As a matter of fact, the Pulse-Wise MVDR is even degrading the data further at some incidence angles because it is blind to the in-swath interference. The orange curve shows the RD-Time MVDR and for the $N=16$ and SNR $=0 \mathrm{~dB}$ only the far range is corrected. When the SNR (saturated curve) or $N$ is increased, the corrupted area around the interferer position gets smaller (due to the longer antenna) and reveals parts of the swath. The RD-Frequency MVDR exhibits the same trend but is reducing the error in between the interferers even for low $N$ and low SNR. When $N$ is increased, the RD-Time MVDR and RD-Frequency MVDR perform comparably (due to the high number of available notches it is not necessary to perform a frequency dependent notching).

The percentage of the recovered swath in dependency of $N$ is plotted from Figure 32c through Figure 32e for the phase standard deviation, the phase offset and the amplitude offset, respectively. 
The recovered swath in the figures has a phase standard deviation error below $20^{\circ}$, a phase offset below $5^{\circ}$ and an amplitude offset below $0.5 \mathrm{~dB}$. As in scenario $\mathrm{B}$, the lost swath halfens for a doubling of the antenna length. Again, the phase offset is the least sensitive parameter and the gain the most sensitive parameter.

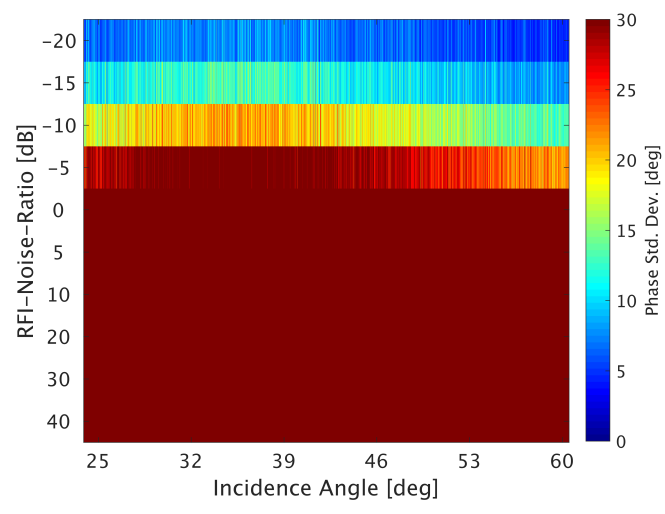

(a)

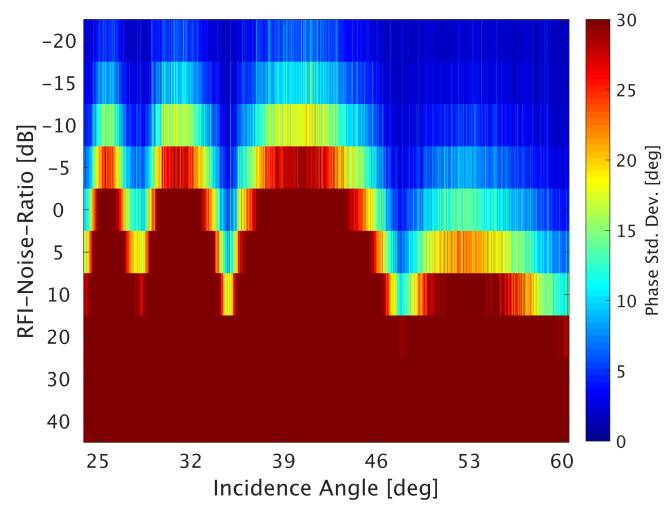

(c)

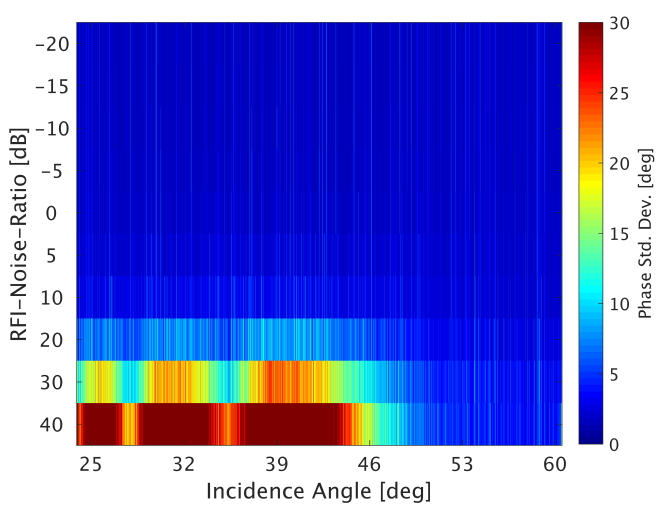

(b)

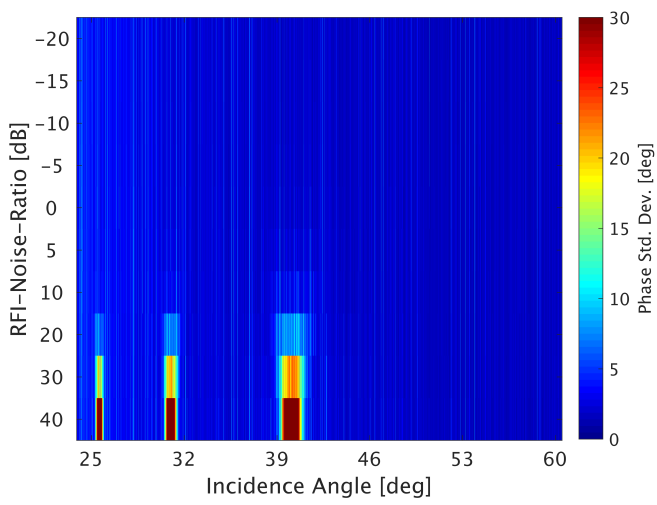

(d)

Figure 31. The phase standard deviation of the residual error image of scenario $D$ for different RFI-Noise-Ratios versus the incidence angle of the instantaneous SAR signal. The SNR is $37.36 \mathrm{~dB}$. (a) No RFI mitigation for $N=8$, (b) After RD-Frequency MVDR beamforming for $N=8$, (c) No RFI mitigation for $N=32$, (d) After RD-Frequency MVDR beamforming for $N=32$. 


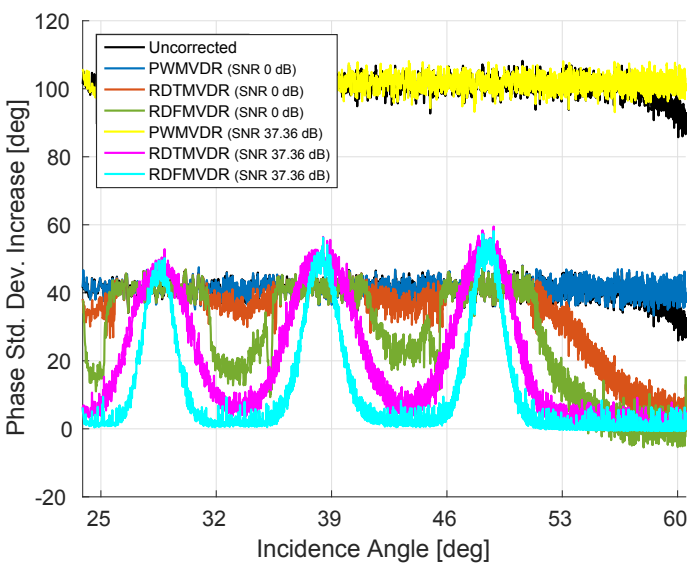

(a)

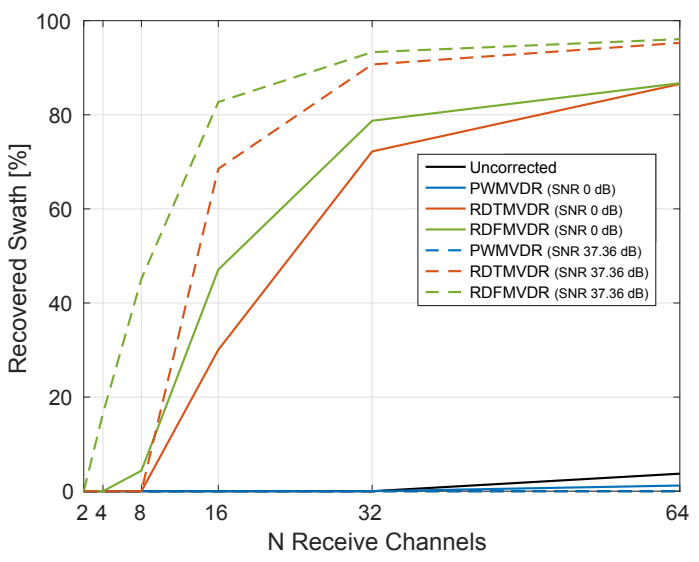

(c)

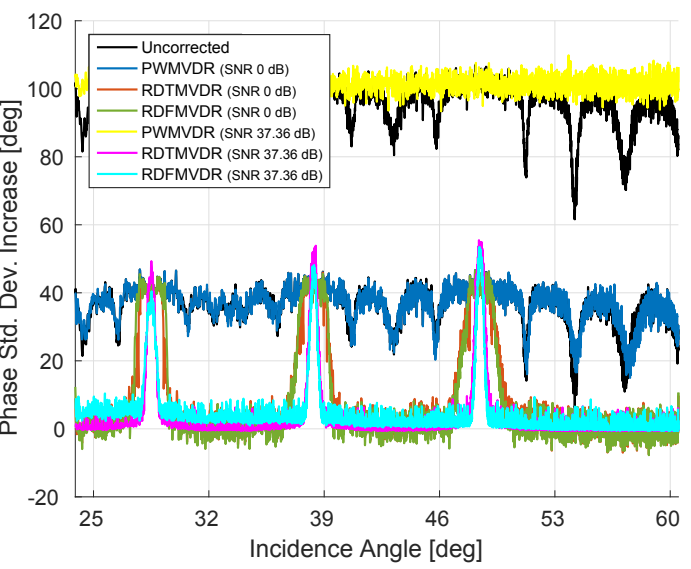

(b)

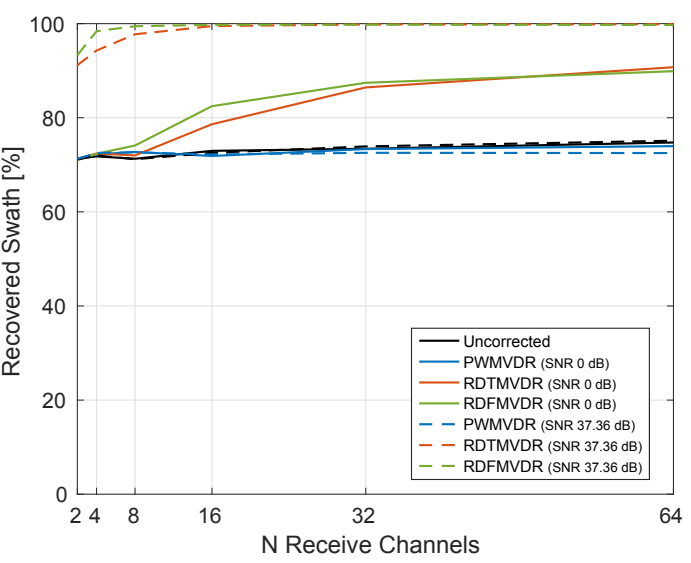

(d)

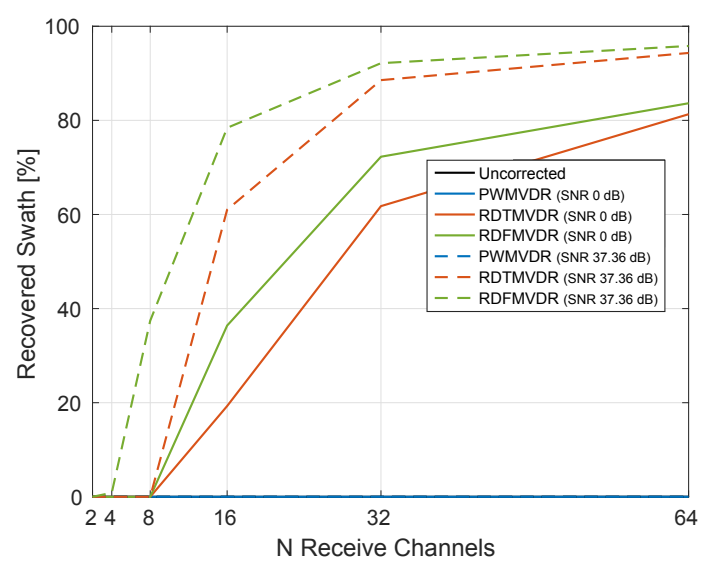

(e)

Figure 32. The phase standard deviation increase of the residual error image for scenario $D$ at a RFI-Noise-Ratio of $40 \mathrm{~dB}$. (a) Error plotted versus incidence angle for $N=16$ and for $\mathrm{SNR}=0 \mathrm{~dB}$ (pale lines) and $\mathrm{SNR}=37.36 \mathrm{~dB}$ (saturated lines), (b) Error plotted versus incidence angle for $N=64$ and for $\mathrm{SNR}=0 \mathrm{~dB}$ (pale lines) and SNR $=37.36 \mathrm{~dB}$ (saturated lines), (c) The percentage of the recovered SAR swath that is within the phase std. dev. limits of $20^{\circ}$, (d) The percentage of the recovered SAR swath that is within the phase offset limits of $5^{\circ}$, (e) The percentage of the recovered SAR swath that is within the absolute gain limits of $0.5 \mathrm{~dB}$. 


\section{Experimental Results}

\subsection{EcoSAR System Description}

EcoSAR is an airborne DBF SAR instrument developed at the NASA Goddard Space Flight Center [21,51-53]. It was funded through the 2010 ESTO Instrument Incubator Program with the goal to measure forest structure, biomass, ice sheets and dry soils. The radar operates at $435 \mathrm{MHz}$ in P-band, the same center frequency that will be used by ESA's BIOMASS mission [54,55], with bandwidths of up to $120 \mathrm{MHz}$ in the horizontal (H) polarization and $200 \mathrm{MHz}$ in the vertical (V) polarization. At this low frequency, the radar benefits from the higher sensitivity of the backscatter to high biomass densities [55]. Furthermore, the temporal decorrelation between repeat passes is reduced due to the increased penetration of the signal and the increased signal return from tree stems and trunks, which are stable over time [56]. The system consists of two dual-polarization antennas that are mounted under both wings of the aircraft as illustrated in Figure 33, allowing it to acquire fully-polarimetric single-pass interferometric data with a baseline of $25 \mathrm{~m}$. Each antenna is composed of eight active subelements that permit DBF in elevation over a range of $\pm 45^{\circ}$. Therefore, the available EcoSAR dataset enables the verification of the algorithms presented in this work on an interferometric SAR system with multiple elevation channels. The performance is assessed by means of the interferometric coherence. For a general overview of EcoSAR, system parameters are listed in the middle column of Table 3 .

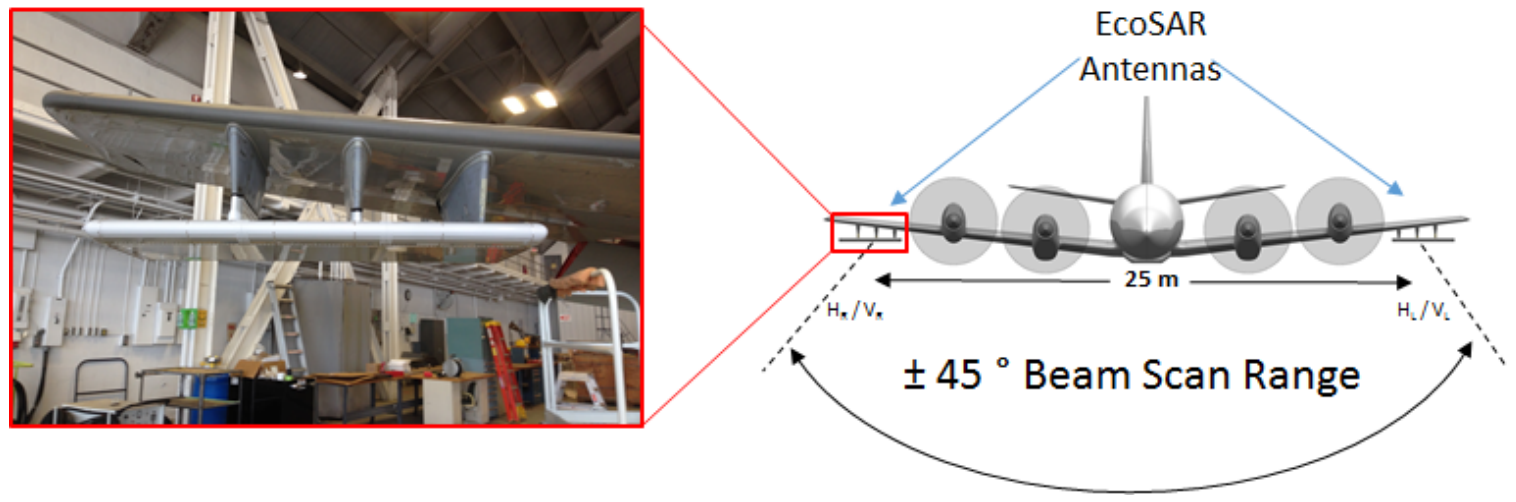

Figure 33. EcoSAR antenna mounted on a wing of the P3 airplane during the 2014 flight campaign (left) and illustration of antenna positions on each wing (right).

Table 3. EcoSAR system parameters: Operational range (middle) and parameters of analyzed dataset (right).

\begin{tabular}{ccc}
\hline & Capability & Dataset \\
\hline Center Frequency & $435 \mathrm{MHz}$ & $479 \mathrm{MHz}$ \\
\hline Bandwidth & $\begin{array}{c}\text { up to } 120 \mathrm{MHz}(\mathrm{H}) \\
\text { up to } 200 \mathrm{MHz}(\mathrm{V})\end{array}$ & $20 \mathrm{MHz}$ \\
\hline Pulse Length & $1 \mu$ s to $50 \mu \mathrm{s}$ & $\begin{array}{c}2.5 \mu \mathrm{s}(\mathrm{H}) \\
1.5 \mu \mathrm{s}(\mathrm{V})\end{array}$ \\
\hline PRF & $100 \mathrm{~Hz}$ to $10 \mathrm{kHz}$ & $500 \mathrm{~Hz}$ \\
\hline Range Resolution & $0.75 \mathrm{~m}$ & $7.5 \mathrm{~m}$ \\
\hline Azimuth Resolution & $0.5 \mathrm{~m}$ & $0.675 \mathrm{~m}$ \\
\hline Array Power & $40 \mathrm{Watts}$ & $40 \mathrm{Watts}$ \\
\hline Flight Altitude & & $3.7 \mathrm{~km}$ \\
\hline Platform Velocity & & $136 \mathrm{~m} / \mathrm{s}$ \\
\hline Physical Baseline & & $25 \mathrm{~m}$ \\
\hline Antenna elements & & $8 \mathrm{per}$ antenna \\
\hline Antenna element spacing & & $0.29 \mathrm{~cm}(0.46 \lambda)$ \\
\hline
\end{tabular}




\subsection{Dataset Description}

From 29 March to 31 March 2014, EcoSAR flew its first flight campaign over Corcovado National Park in Costa Rica. EcoSAR was operating in dual-track mode, illuminating both sides of the aircraft with a wide transmit beam. This allowed the simultaneous acquisition of both sides of the flight track, effectively doubling the swath. Due to EcoSAR's DBF capability, it was then possible to electronically steer the receive beam to both sides of the track in post-processing. The ground track of all flight lines is shown in Figure 34 on the left. Green and red boxes represent the swath on the left and right side of the airplane respectively. As shown, four different flight lines were flown over Corcovado. However, due to the complexity of the system, there were synchronization issues among the receive channels that appeared in flight, which were undetectable in the lab. These included time delays and phase offsets in range and azimuth between the channels. The range dependent errors changed abruptly every few minutes, causing about one change within a recorded flight line. A change of the azimuth dependent errors occured every few seconds within a flight line and can be contributed to missing pulses in the RAW data. These error sources have now been repaired for future flights and the previously collected data has largely been corrected. Nevertheless, this gave rise to a degraded DBF performance. Additionally, synchronization issues between the receive windows of each antenna and polarization, as well as a too short receive window, decreased the common spectral overlap between interferometric pairs. The processed range bandwidth therefore needed to be reduced to $20 \mathrm{MHz}$, with only $2.5 \mu$ s of the pulse recovered for the $\mathrm{H}$ polarzation ( $1.5 \mu \mathrm{s}$ for the $\mathrm{V}$ polarization). This affected the SNR and resulted in noisy interferograms. In addition, the transmit power of the $\mathrm{V}$ polarization was lower than intended, further decreasing the SNR of interferograms that utilize the V polarization in transmit. After all error sources were corrected, only the flight line Osa_T1 (Figure 34) was of good enough quality to produce interferograms. The geocoded $\mathrm{HH}$ image of this flight line is shown in Figure 34 on the right. Note that the shown image covers the entire processed bandwidth of $120 \mathrm{MHz}$ for the $\mathrm{H}$ polarization and that the swath size in range is decreased for the interferograms. This is the case because SAR interferometry requires to filter the images to a mutual bandwidth, which avoids a degradation of the interferometric coherence. Because of the timing issues between the antennas and because of the cropped receive window, this resulted in a frequency overlap between the images of only $20 \mathrm{MHz}$.

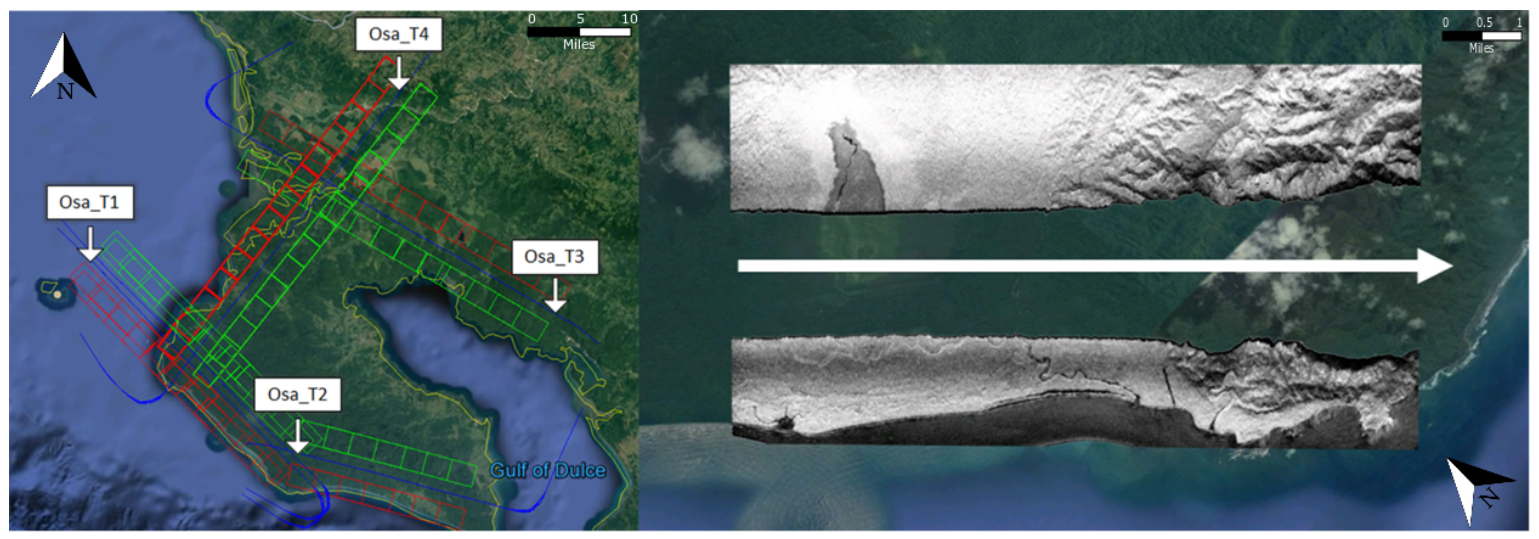

Figure 34. Flight lines of the collected EcoSAR data in Costa Rica in March 2014. Green and red boxes represent the image swaths on left and right respectively (left). Geocoded EcoSAR HH image of Osa_T1 analysed in this section. Data was acquired on both sides of the flight track simultaneously (right).

\subsection{Results}

In this section, the EcoSAR interferograms are compared before and after RFI mitigation with DBF. Note that only a selection of the algorithms presented in Section 3 are applied for the following reasons: (a) EcoSAR only acquired data with DBF in elevation, (b) Range-dependent ambiguities are affecting the dual-track mode data of EcoSAR as discussed below. (c) Figure 35a shows the averaged frequency 
spectrum of the HH and VHimage. Multiple peaks due to narrowband RFI are clearly visible in the right half of the SAR spectrum which indicates continous RFI. The Pulsed-RFI MVDR can therefore be ruled out.

EcoSAR is only equipped with eight receive channels per antenna, thus 7 notches can be placed towards interference. If the entire bandwidth was used for the interferograms, it would be recommended to apply the RFI filtering on multiple sublooks to make best use of the number of available notches. However, Figure $35 \mathrm{~b}$ shows the part relevant to the interferograms that are filtered to a common bandwidth with the spectrum ranging from $469 \mathrm{MHz}$ to $489 \mathrm{MHz}$. In the processed bandwidth only three peaks can be made out in the $\mathrm{HH}$ and five peaks in the $\mathrm{VH}$ image. Note that AoA estimations on the EcoSAR data can be found in Reference [57]. The dual-track mode of EcoSAR doubles the covered swath, though this makes it necessary to reserve notches for ambiguity suppression to suppress signals from the opposite side. These ambiguities are handled by the RD-Time MVDR/RD-Frequency MVDR as if they were interferences and no modification of the algorithms are needed. However, the ability to notch external interferences is reduced compared to single-track mode. Because about three notches are required to suppress these ambiguities, this leaves four notches for interference. Therefore, the HH image should show a good RFI filter behaviour as more notches than interferers are available. The $\mathrm{VH}$ image on the other hand has not enough notches available and the filter capabilities might be reduced. In addition, the power (and hence SNR) in the VH image is weaker than the HH image, partially due to the weaker transmit power and partially because the power is spread over a higher total bandwidth (H: $120 \mathrm{MHz}, \mathrm{V}: 200 \mathrm{MHz})$ even though only $20 \mathrm{MHz}$ are processed. Overall, the total coherence and the improvement due to the RFI mitigation is expected to be higher for the $\mathrm{HH}$ interferogram.

Computed $\mathrm{HH}$ and $\mathrm{VH}$ coherence histograms for both sides are plotted in Figure 36. The blue curve represents uncorrected data and shows the worst coherence in all four cases. It can be noticed that the improvement for the left side (Figure 36a,c) is higher than for the right side (Figure 36b,d). A possible explanation for this is, that external interferers are located on the left side of the track and are already suppressed well by the sidelobes when the beam is steered towards the right side. This also seems logical because the right side is looking towards the sea, where the likelihood for interference is expected to be lower. The performance of the RD-Time MVDR and RD-Frequency MVDR is similar, making the faster RD-Time MVDR the better choice. The better performance of the RD-Frequency MVDR in the simulations can be contributed to the larger bandwidth (120 MHz). Because the processed bandwidth of the available dataset is only $20 \mathrm{MHz}$, the frequency resolution of the RD-Frequency MVDR can not utilize its full potential. The coherence gains are also higher compared to the manual notching of the ambiguities. In Figure 36b,c, the tail ends of the manual notching approach reaches into higher coherences but the overall coherence values are improved with the automated methods. Possible reasons for this include the high coherence seen in the mountains. As the MVDR beamformer is sensitive to a wrong steering direction of the main beam, a degradation is caused by the filter and hence the SNR drops. A solution for this would be an additional constraint to widen the main beam (at the cost of one notch) or to implement a topography-dependent steering rather than assuming a flat earth model. 


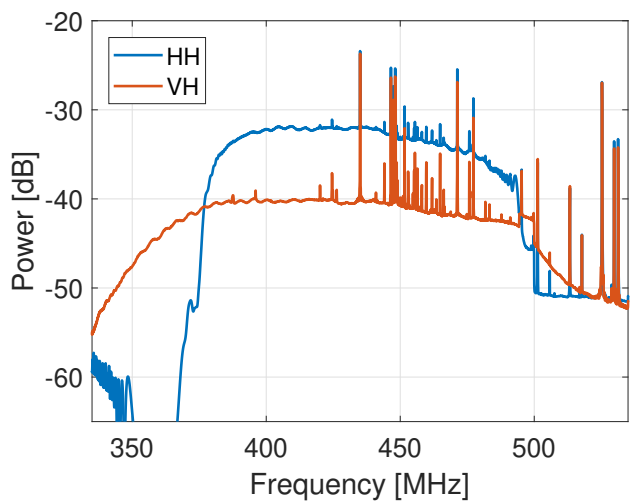

(a)

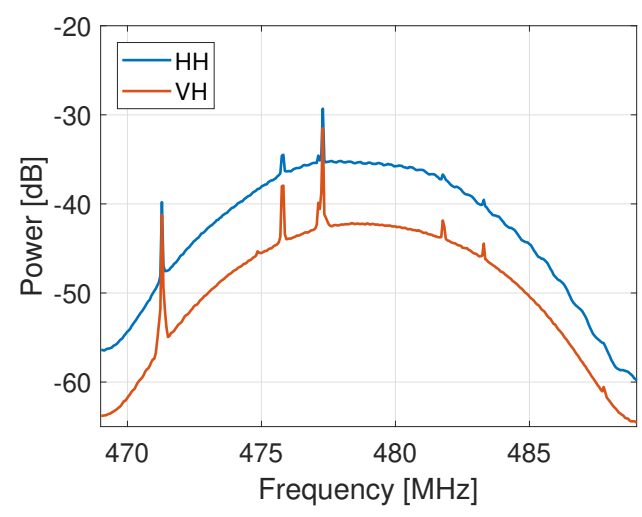

(b)

Figure 35. Averaged range-frequency spectrum of the available EcoSAR scene for the $\mathrm{HH}$ (blue) and VH (orange) image: (a) Entire recorded spectrum with cut-off chirp due to too short receive window; (b) Spectrum after hamming window is applied to keep common spectrum only.

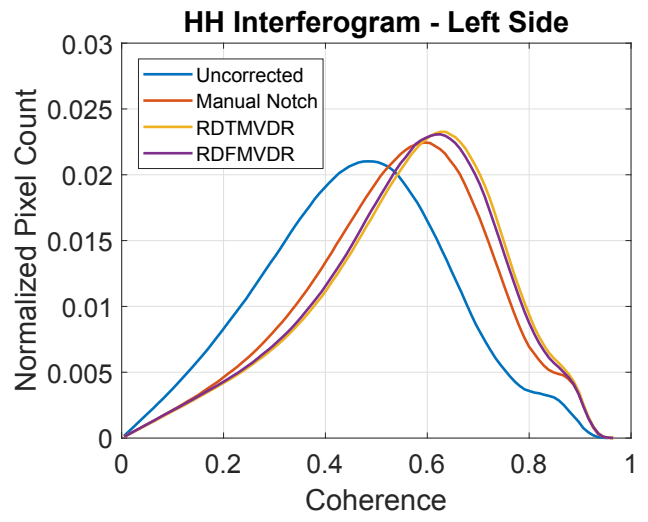

(a)

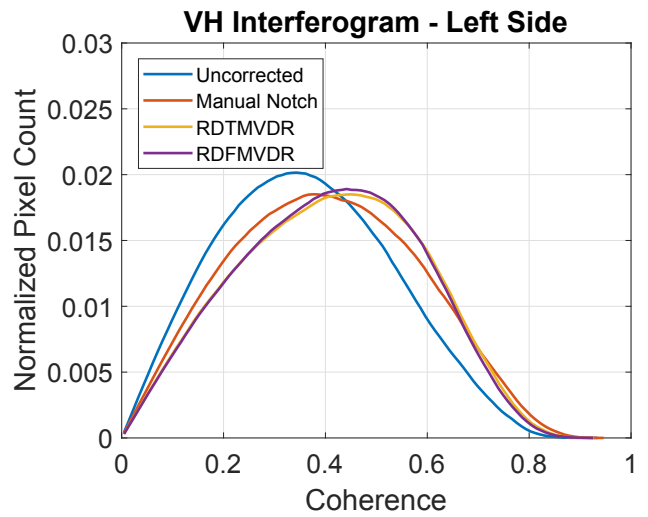

(c)

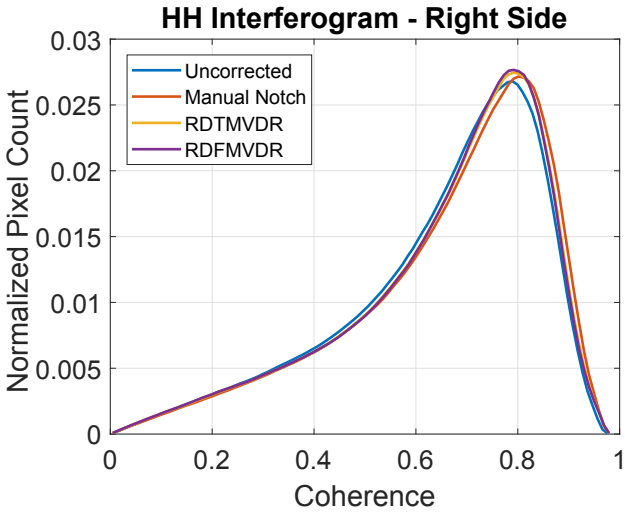

(b)

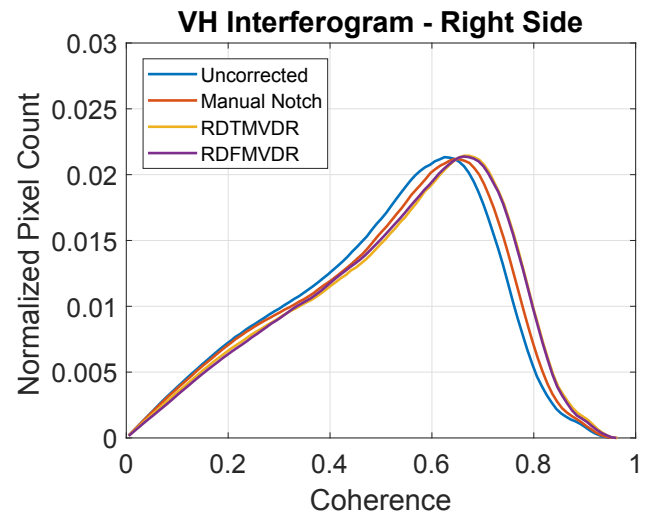

(d)

Figure 36. Coherence Histograms for uncorrected, manually notched, RD-Time MVDR filtered and RD-Frequency MVDR filtered image for (a) HH interferogram of left side, (b) HH interferogram of right side, (c) VH interferogram of left side, (d) VH interferogram of right side. The normalized pixel count values reported on the left axis indicate the ratio of the number of pixels with the given coherence value to the total number of pixels.

Coherence images for the $\mathrm{HH}$ interferogram of the left side, which showed the biggest improvement according to the histograms, are shown in Figure 37. The improvement from the uncorrected image (second from top) to the manually notched image (third from top) is evident and marked with red boxes. Periodic coherence losses in the middle of both images could be caused 
by the narrow band interferences observed in the spectrum of Figure 35b. After implementing the RD-Time MVDR (bottom row), the periodic coherence losses vanish, producing a clearer coherence image with higher coherence values.
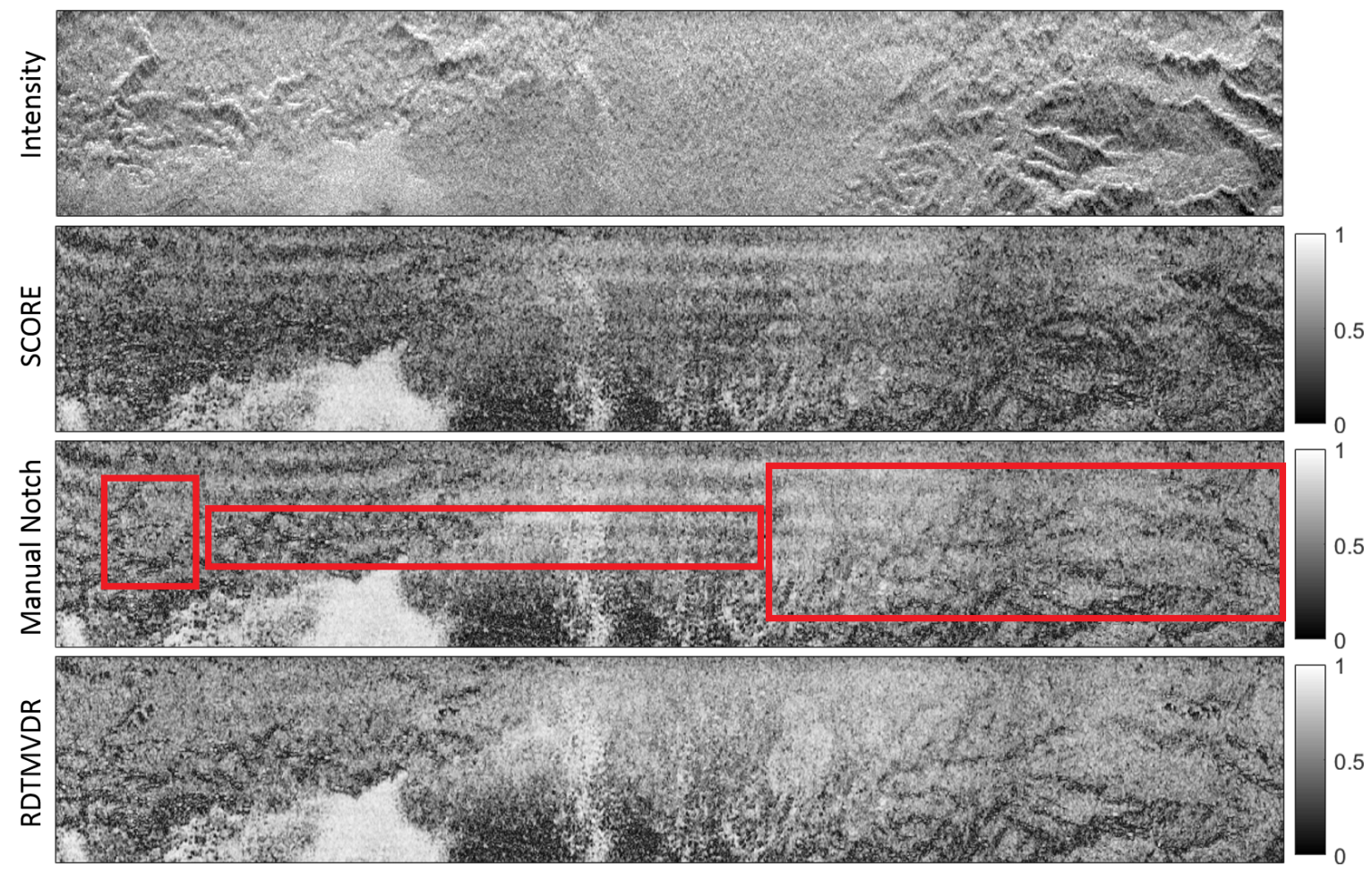

Figure 37. Top to bottom: Intensity of focused SAR image, HH coherence with Scan-On-Receive and $\mathrm{HH}$ coherence with adaptive RD-Time MVDR for ambiguity and RFI suppression. Periodic coherence losses due to interference are visible in the uncorrected image and in the manually notched image. Red boxes mark areas of most evident coherence improvement after notching ambiguities from the other side. After the RFI mitigation, the periodic coherence losses disappear.

\section{Conclusions}

In this paper, we presented new RFI mitigations methods for SAR based on DBF in elevation and/or azimuth. By utilizing the spatial distribution of the SAR signal in the range-Doppler domain, it is possible to adaptively place antenna pattern notches throughout the image for an optimized filtering. In the absence of in-swath interference and if there are more notches available than interferers (large N), the Pulse-Wise MVDR performs best. This is because the RD-Time MVDR and RD-Frequency MVDR introduce a larger processing noise. However, if there are in-swath interferers (or more interferers than notches available) the Pulse-Wise MVDR fails. In that case, the range-dependent algorithms can be applied at the cost of an increased processing power. The simulation showed that the RD-Frequency MVDR introduces a higher processing noise than the RD-Time MVDR but the RD-Frequency MVDR is also capable to operate at smaller $N$ (because the notches are set frequency-dependent, which comes at the expense of processing time). The advantage of the RD-Frequency MVDR decreases with increasing $N$. It is therefore recommended to use the RD-Frequency MVDR for receive antennas with small $N$ or if many interferers are expected. Otherwise, the RD-Time MVDR performs best. In regard to $N$ it should also be mentioned that many close interferers can be notched with few $N$ because they can be suppressed with the same wide notch. In theory, a single notch is sufficient to notch one interferer but relying on one notch makes the notching sensitive to AoA errors of the RFI. Placing wider notches ensures that the interferer is suppressed well and is beneficial for strong interferers. As predicted, in-swath interference can not be removed with antenna pattern notching when the instantaneous main (SCORE) beam looks into 
the interferer direction. However, the lost swath halfens for a doubling of the antenna length, which is due to the resulting halfening of the beamwidth. In the end, RFI mitigation based on time and frequency adaptive antenna pattern notching was applied to EcoSAR imagery and first results were presented. Even though the EcoSAR data was corrupted due to hardware glitches and affected by uncalibrated channels, the applied techniques showed a good improvement of the coherence. Further, ambiguities from the opposite side of the flight track caused by a dual-track imaging mode were treated as interference and handled well by the algorithm. This concludes that the range-dependent algorithms are also able to handle an automatic notching of, for example, orthogonal waveforms in MIMO-SAR [58,59] or multiple elevation beams [58]. The manual notching of these ambiguities is sensitive to inaccuracies of the assumed signal AoAs $[35,60]$ (e.g., due to topography). The notches were adaptively driven towards the ambiguity and towards external interference for the best result in each range line. A further improvement of the RFI filtering is expected from uncorrupted and calibrated DBF data that could be acquired with a new EcoSAR flight campaign.

Author Contributions: T.B. generated the results, developed the algorithms presented in the paper and processed the EcoSAR images. B.O. assisted in algorithm development. B.O., S.-K.L., and R.R. assisted with the EcoSAR data collection and image processing. S.-K.L., and T.F. contributed to the interpretation of the results and helped in assessing the performance of the algorithms.

Funding: This research was funded by NASA Earth Science Technology Office funding 10-IIP10-0060, and Goddard Space Flight Center 2019 Internal Research and Development Award 113.

Conflicts of Interest: The authors declare no conflict of interest.

\section{References}

1. Mazar, H. Radio Spectrum Management: Policies, Regulations and Techniques; John Wiley \& Sons: Somerset, UK, 2016. [CrossRef]

2. Radio Regulations; ITU: Geneva, Switzerland, 2016.

3. ITU-R. Recommendation ITU-R RS.1260-1. Feasibility of Sharing Between Active Spaceborne Sensors and Other Services in the Range 420-470 MHz; International Telecommunication Union: Geneva, Switzerland, 2003.

4. Shimada, M.; Isoguchi, O.; Tadono, T.; Isono, K. PALSAR Radiometric and Geometric Calibration. IEEE Trans. Geosci. Remote Sens. 2009, 47, 3915-3932. [CrossRef]

5. Rosen, P.A.; Hensley, S.; Le, C. Observations and mitigation of RFI in ALOS PALSAR SAR data: Implications for the DESDynI mission. In Proceedings of the 2008 IEEE Radar Conference, Rome, Italy, 26-30 May 2008; pp. 1-6. [CrossRef]

6. Meyer, F.J.; Nicoll, J.B.; Doulgeris, A.P. Correction and Characterization of Radio Frequency Interference Signatures in L-Band Synthetic Aperture Radar Data. IEEE Trans. Geosci. Remote Sens. 2013, 51, 4961-4972. [CrossRef]

7. Lord, R.T.; Inggs, M.R. Approaches to RF interference suppression for VHF/UHF synthetic aperture radar. In Proceedings of the 1998 South African Symposium on Communications and Signal Processing, Rondebosch, South Africa, 8 September 1998; pp. 95-100. [CrossRef]

8. Chang, J.L.; Li, X. The Effect of Notch Filter on RFI Suppression. Wirel. Sens. Netw. 2009, 1, $196-205$. [CrossRef]

9. Reigber, A.; Ferro-Famil, L. Interference suppression in synthesized SAR images. IEEE Geosci. Remote Sens. Lett. 2005, 2, 45-49. [CrossRef]

10. Potsis, A.; Reigber, A.; Papathanassiou, K.P. A phase preserving method for RF interference suppression in P-band synthetic aperture radar interferometric data. In Proceedings of the IGARSS '99 IEEE International Geoscience and Remote Sensing Symposium, Hamburg, Germany, 28 June-2 July 1999; Volume 5, pp. 2655-2657. [CrossRef]

11. Braunstein, M.; James, M.; Ralston, D.A.S. Signal processing approaches to radio frequency interference (RFI) suppression. In Proceedings of the SPIE's International Symposium on Optical Engineering and Photonics in Aerospace Sensing, Orlando, FL, USA, 4-8 April 1994; Volume 2230.

12. Lord, R.T.; Inggs, M.R. Efficient RFI suppression in SAR using LMS adaptive filter integrated with range/Doppler algorithm. Electron. Lett. 1999, 35, 629-630. [CrossRef] 
13. Le, C.T.C.; Hensley, S.; Chapin, E. Removal of RFI in wideband radars. In Proceedings of the IGARSS '98 IEEE International Geoscience and Remote Sensing Symposium Proceedings, Seattle, WA, USA, 6-10 July 1998; Volume 4, pp. 2032-2034. [CrossRef]

14. Applebaum, S. Adaptive arrays. IEEE Trans. Antennas Propag. 1976, 24, 585-598. [CrossRef]

15. Farina, A. Single Sidelobe Canceller: Theory and Evaluation. IEEE Trans. Aerosp. Electron. Syst. 1977, AES-13, 690-699. [CrossRef]

16. Farina, A. Evaluation of sidelobe-canceller performance. Commun. Radar Signal Process. IEE Proc. F 1982, 129, 52-58. [CrossRef]

17. Sedehi, M.; Cristallini, D.; Marini, J.; Lombardo, P. Impact of an Electromagnetic Interference on Imaging Capability of a Synthetic Aperture Radar. In Proceedings of the 2007 IEEE Aerospace Conference, Big Sky, MT, USA, 3-10 March 2007; pp. 1-8. [CrossRef]

18. Sedehi, M.; Colone, F.; Cristallini, D.; Lombardo, P. A reduced order jammer cancellation scheme based on double adaptivity. In Proceedings of the 2008 IEEE Radar Conference, Rome, Italy, 26-30 May 2008; pp. 1-6. [CrossRef]

19. Lombardo, P.; Sedehi, M.; Colone, F.; Bucciarelli, M.; Cristallini, D. Dual Channel Adaptive Antenna Nulling with Auxiliary Selection for Spaceborne Radar. In Proceedings of the 2008 IEEE Aerospace Conference, Big Sky, MT, USA, 1-8 March 2008; pp. 1-8. [CrossRef]

20. Sedehi, M.; Cristallini, D.; Bucciarelli, M.; Lombardo, P. Constrained adaptive beamforming for electromagnetic interference cancellation for a synthetic aperture radar. In Proceedings of the IET International Conference on Radar Systems, Edinburgh, UK, 15-18 October 2007; pp. 1-5. [CrossRef]

21. Rincon, R.F.; Fatoyinbo, T.; Osmanoglu, B.; Lee, S.; Ranson, K.J.; Sun, G.; Bollian, T. ECOSAR: P-Band Digital Beamforming Polarimetric and Single Pass Interferometric SAR: Instrument Performance. In Proceedings of the EUSAR 2016: 11th European Conference on Synthetic Aperture Radar, Hamburg, Germany, 6-9 June 2016; pp. 1-5.

22. Rincon, R.F.; Fatoyinbo, T.; Ranson, J.; Sun, G.; Perrine, M.; Bonds, Q.; Valett, S.; Seufert, S. Digital Beamforming Synthetic Aperture Radar (DBSAR) polarimetric operation during the Eco3D flight campaign. In Proceedings of the IEEE International Geoscience and Remote Sensing Symposium, Munich, Germany, 22-27 July 2012; pp. 1549-1552. [CrossRef]

23. Younis, M.; Huber, S.; Herrero, C.T.; Krieger, G.; Moreira, A.; Uematsu, A.; Sudo, Y.; Nakamura, R.; Chishiki, Y.; Shimada, M. Tandem-L instrument design and SAR performance overview. In Proceedings of the 2014 IEEE Geoscience and Remote Sensing Symposium, Quebec City, QC, Canada, 13-18 July 2014; pp. 88-91. [CrossRef]

24. Younis, M.; Fischer, C.; Wiesbeck, W. Digital beamforming in SAR systems. IEEE Trans. Geosci. Remote Sens. 2003, 41, 1735-1739. [CrossRef]

25. Veen, B.D.V.; Buckley, K.M. Beamforming: A versatile approach to spatial filtering. IEEE ASSP Mag. 1988, 5, 4-24. [CrossRef]

26. Suess, M.; Grafmueller, B.; Zahn, R. A novel high resolution, wide swath SAR system. In Proceedings of the IGARSS 2001, Scanning the Present and Resolving the Future, IEEE 2001 International Geoscience and Remote Sensing Symposium (Cat. No.01CH37217), Sydney, NSW, Australia, 9-13 July 2001; Volune 3, pp. 1013-1015. [CrossRef]

27. Suess, M.; Wiesbeck, W. Side-looking Synthetic Aperture Radar System. European Patent EP 1241487 B1, 18 September 2006.

28. Capon, J. High-resolution frequency-wavenumber spectrum analysis. Proc. IEEE 1969, 57, 1408-1418. [CrossRef]

29. Trees, H. Optimum Array Processing (Detection, Estimation, and Modulation Theory, Part IV); Wiley-Interscience: New York, NY, USA, 2002.

30. Lorenz, R.G.; Boyd, S.P. Robust minimum variance beamforming. IEEE Trans. Signal Process. 2005, 53, 1684-1696. [CrossRef]

31. Cox, H. Resolving power and sensitivity to mismatch of optimum array processors. J. Acoust. Soc. Am. 1973, 54, 771-785. [CrossRef]

32. Vural, A. Effects of perturbations on the performance of optimum/adaptive arrays. IEEE Trans. Aerosp. Electron. Syst. 1979, AES-15, 76-87. [CrossRef] 
33. Compton, R. The effect of random steering vector errors in the Applebaum adaptive array. IEEE Trans. Aerospa. Electron. Syst. 1982, AES-18, 392-400. [CrossRef]

34. Wax, M.; Anu, Y. Performance analysis of the minimum variance beamformer in the presence of steering vector errors. IEEE Trans. Signal Process. 1996, 44, 938-947. [CrossRef]

35. Bordoni, F.; Younis, M.; Varona, E.M.; Krieger, G. Adaptive scan-on-receive based on spatial spectral estimation for high-resolution, wide-swath Synthetic Aperture Radar. In Proceedings of the IEEE International Geoscience and Remote Sensing Symposium, Cape Town, South Africa, 12-17 July 2009; Volume 1, pp. I-64-I-67.

36. Frost, O.L. An algorithm for linearly constrained adaptive array processing. Proc. IEEE 1972, 60, 926-935. [CrossRef]

37. Sedehi, M.; Bucciarelli, M.; Cristallini, D.; Scolamiero, S.; Lombardo, P. Interference covariance matrix estimation for a Multi-Channel Synthetic Aperture Radar. In Proceedings of the 7th European Conference on Synthetic Aperture Radar, Friedrichshafen, Germany, 2-5 June 2008; pp. 1-4.

38. Villano, M.; Krieger, G.; Moreira, A. Staggered SAR: High-resolution wide-swath imaging by continuous PRI variation. IEEE Trans. Geosci. Remote Sens. 2014, 52, 4462-4479. [CrossRef]

39. Villano, M.; Krieger, G.; Moreira, A. A novel processing strategy for staggered SAR. IEEE Geosci. Remote Sens. Lett. 2014, 11, 1891-1895. [CrossRef]

40. Villano, M.; Krieger, G.; Jäger, M.; Moreira, A. Staggered SAR: Performance Analysis and Experiments with Real Data. IEEE Trans. Geosci. Remote Sens. 2017, 55, 6617-6638. [CrossRef]

41. De Almeida, F.Q.; Younis, M.; Krieger, G.; Moreira, A. Multichannel staggered SAR azimuth processing. IEEE Trans. Geosci. Remote Sens. 2018, 56, 2772-2788. [CrossRef]

42. Gu, Y.; Leshem, A. Robust adaptive beamforming based on interference covariance matrix reconstruction and steering vector estimation. IEEE Trans. Signal Process. 2012, 60, 3881-3885.

43. Younis, M.; Rommel, T.; Bordoni, F.; Krieger, G.; Moreira, A. On the pulse extension loss in digital beamforming SAR. IEEE Geosci. Remote Sens. Lett. 2015, 12, 1436-1440. [CrossRef]

44. Moreira, A. Radar Mit Synthetischer Apertur; Oldenbourg Verlag: München, Germany, 1999.

45. Schmidt, R. Multiple emitter location and signal parameter estimation. IEEE Trans. Antennas Propag. 1986, 34, 276-280. [CrossRef]

46. Wu, H.T.; Yang, J.F.; Chen, F.K. Source number estimators using transformed Gerschgorin radii. IEEE Trans. Signal Process. 1995, 43, 1325-1333. [CrossRef]

47. Qiu, Z.; He, Z.; Cui, M. A method for signal source number estimation based on MUSIC algorithm. In Proceedings of the IEEE International Conference on Communication Problem-Solving (ICCP), Guilin, China, 16-18 October 2015; pp. 344-346. [CrossRef]

48. Rapuano, S.; Harris, F.J. An introduction to FFT and time domain windows. IEEE Instrum. Meas. Mag. 2007, 10, 32-44. [CrossRef]

49. Yegulalp, A.F. Fast backprojection algorithm for synthetic aperture radar. In Proceedings of the 1999 IEEE Radar Conference. Radar into the Next Millennium (Cat. No.99CH36249), Waltham, MA, USA, 22-22 April 1999; pp. 60-65. [CrossRef]

50. Duersch, M.I. Backprojection for Synthetic Aperture Radar; Brigham Young University: Provo, UT, USA, 2013.

51. Rincon, R.F.; Fatoyinbo, T.; Ranson, K.J.; Osmanoglu, B.; Sun, G.; Deshpande, M.; Perrine, M.; Toit, C.D.; Bonds, Q.; Beck, J.; et al. The ecosystems SAR (EcoSAR) an airborne P-band polarimetric InSAR for the measurement of vegetation structure, biomass and permafrost. In Proceedings of the IEEE Radar Conference, Cincinnati, OH, USA, 19-23 May 2014; pp. 1443-1445. [CrossRef]

52. Rincon, R.F.; Fatoyinbo, T.; Osmanoglu, B.; Lee, S.; Ranson, K.J.; Sun, G.; Perrine, M.; Toit, C.D. ECOSAR: P-band digital beamforming polarimetric and single pass interferometric SAR. In Proceedings of the IEEE Radar Conference (RadarCon), Arlington, VA, USA, 10-15 May 2015; pp. 0699-0703. [CrossRef]

53. Osmanoglu, B.; Rincon, R.; Lee, S.; Bollian, T.; Fatoyinbo, T. Forest structure retrieval from EcoSAR P-band single-pass interferometry. In Proceedings of the IEEE International Geoscience and Remote Sensing Symposium (IGARSS), Fort Worth, TX, USA, 23-28 July 2017; pp. 4296-4305. [CrossRef]

54. Carreiras, J.M.; Quegan, S.; Toan, T.L.; Minh, D.H.T.; Saatchi, S.S.; Carvalhais, N.; Reichstein, M.; Scipal, K. Coverage of high biomass forests by the ESA BIOMASS mission under defense restrictions. Remote Sens. Environ. 2017, 196, 154-162. [CrossRef] 
55. Toan, T.L.; Quegan, S.; Davidson, M.; Balzter, H.; Paillou, P.; Papathanassiou, K.; Plummer, S.; Rocca, F.; Saatchi, S.; Shugart, H.; et al. The BIOMASS mission: Mapping global forest biomass to better understand the terrestrial carbon cycle. Remote Sens. Environ. 2011, 115, 2850-2860. [CrossRef]

56. Dubois-Fernandez, P.C.; Toan, T.L.; Daniel, S.; Oriot, H.; Chave, J.; Blanc, L.; Villard, L.; Davidson, M.W.J.; Petit, M. The TropiSAR Airborne Campaign in French Guiana: Objectives, Description, and Observed Temporal Behavior of the Backscatter Signal. IEEE Trans. Geosci. Remote Sens. 2012, 50, 3228-3241. [CrossRef]

57. Bollian, T.; Osmanoglu, B.; Rincon, R.F.; Lee, S.; Fatoyinbo, T.E. Detection and Geolocation ofP-Band Radio Frequency Interference Using EcoSAR. IEEE J. Sel. Top. Appl. Earth Obs. Remote Sens. 2018, 11, 3608-3616. [CrossRef]

58. Krieger, G.; Gebert, N.; Moreira, A. Multidimensional Waveform Encoding: A New Digital Beamforming Technique for Synthetic Aperture Radar Remote Sensing. IEEE Trans. Geosci. Remote Sens. 2008, 46, 31-46. [CrossRef]

59. Krieger, G. MIMO-SAR: Opportunities and Pitfalls. IEEE Trans. Geosci. Remote Sens. 2014, 52, $2628-2645$. [CrossRef]

60. Krieger, G.; Huber, S.; Villano, M.; Younis, M.; Rommel, T.; Dekker, P.L.; de Almeida, F.Q.; Moreira, A. CEBRAS: Cross elevation beam range ambiguity suppression for high-resolution wide-swath and MIMO-SAR imaging. In Proceedings of the IEEE International Geoscience and Remote Sensing Symposium (IGARSS), Milan, Italy, 26-31 July 2015; pp. 196-199. [CrossRef]

(C) 2019 by the authors. Licensee MDPI, Basel, Switzerland. This article is an open access article distributed under the terms and conditions of the Creative Commons Attribution (CC BY) license (http://creativecommons.org/licenses/by/4.0/). 